\title{
Clusterorganisationen in der Praxis
}

\section{Strukturelle und institutionelle Einflussfaktoren auf eine clustertheoretisch orientierte Wirtschaftsförderung}

\author{
Karl Häfner ${ }^{1}$
}

Eingegangen: 18. Dezember 2015 / Angenommen: 28. Juli 2016 / Online publiziert: 9. August 2016

(C) Springer-Verlag Berlin Heidelberg 2016

Zusammenfassung Clusterorganisationen setzen Clusterpolitik in die Praxis um und prägen ihre Wirkung damit in hohem Maße. Der Handlungsspielraum der Clusterorganisationen wird dabei stark durch Interaktionen mit den zu fördernden Unternehmen, der Politik und weiteren Förderakteuren beeinflusst. Für eine fundierte Beratung der Clusterförderung sollte man diese Einflussfaktoren kennen und ernst nehmen, da sie die Stärken, Schwächen und Potenziale der Clusterorganisationen mitgestalten. Dieser Beitrag untersucht, wie bayerische Clusterorganisationen die Clustertheorien in praktische Aktivitäten umsetzen und wie sie dabei durch andere Akteure in ihrem Umfeld beeinflusst werden. Dabei wird zwischen institutionellen und strukturellen Einflussfaktoren unterschieden. Institutionelle Einflussfaktoren beziehen sich auf soziale Institutionen, die andere Akteure an die Clusterorganisationen herantragen. Es wird gezeigt, dass die Akteure die Arbeit der Clusterorganisationen zwar in hohem Maße prägen, aber nur in wenigen Fällen ernsthaft behindern. Position und Aufgaben der Clusterorganisationen innerhalb des gesamten Fördersystems erzeugen strukturelle Einflussfaktoren. Diese führen dazu, dass die Clusterorganisationen jenseits des Aufbaus von clusterinternen Netzwerken nur eingeschränkte Handlungsmöglichkeiten haben und für eine umfassende Clusterförderung auf Kooperationen mit anderen Förderakteuren angewiesen sind.

Schlüsselwörter Clusterpolitik · Clusterorganisationen · Wirtschaftsförderung · Bayern

Dr. Karl Häfner

karl.haefner@uni-tuebingen.de

1 Forschungsbereich Geographie, Eberhard-Karls-Universität Tübingen, Rümelinstr. 19-23, 72070 Tübingen, Deutschland

\section{Cluster Organizations in Praxis}

Structural and institutional influences on a cluster-orientated promotion of economic development

Abstract Cluster organizations implement cluster policies and affect the outcome of them to a high degree. Their work is influenced by interactions with the promoted firms, political and other actors. A sound consultancy of cluster promotion should know these influences and take them seriously, because they shape the strengths, weaknesses and potentials of cluster organizations. This article examines how cluster organizations in Bavaria translate cluster theory into practical activities and how other actors influence them in the process. The influences are subdivided into institutional and structural factors. Institutional factors refer to social institutions that other actors impose on the organizations. It will be shown that these factors shape the work of cluster organizations to a high degree, but only seldom restrain it. The organizations positions and tasks within the wider economic promotion system constitute the structural factors. They confine the organizations towards networking activities and force them to cooperate with other promotional actors, if they want to provide a comprehensive cluster promotion.

Keywords Cluster policy · Cluster organizations · Regional economic development · Bavaria

\section{Einleitung}

Das Clusterkonzept ist in der regionalen Wirtschaftsförderung ungebrochen populär und ein Ende des Trends ist vorerst nicht absehbar (Koschatzky 2012: 201; Beck/ Heinze/Schmid 2014a: 23, 37). Seit über zwei Dekaden 
nehmen Wirtschaftsförderer jene räumliche Ballungen von Unternehmen und anderen Akteuren aus gleichen oder verwandten Branchen, die Porter (1998a, 1998b) unter dem Begriff „Cluster" populär gemacht hat, zum Ausgangspunkt ihrer Arbeit. Wirtschaftsgeographen verfolgen diese Entwicklung jedoch mit Skepsis. Neben grundsätzlicher (konstruktiver) Kritik an der Clustertheorie auf theoretischer und empirischer Ebene (vgl. z. B. Martin/Sunley 2003) wird immer wieder festgestellt, dass in der Förderpraxis ein stark reduziertes Verständnis der Zusammenhänge in Clustern vorherrscht und die spezifischen lokalen Kontexte zu wenig beachtet werden (Bergman/ Feser 1999; Asheim/Cooke/Martin 2006; Kiese 2008b; Kiese 2012; Benner 2012; Fromhold-Eisebith 2014). Dementsprechend befürchten Beobachter aus der Wissenschaft, dass die Praktiker die Theorie unsachgemäß anwenden, was zu unerwünschten Entwicklungstendenzen in den jeweiligen Regionen führen kann. Verschiedene Autoren plädieren daher für eine stärkere Auseinandersetzung der wissenschaftlichen Geographie mit der clusterorientierten Wirtschaftsförderung, um die Potenziale und Gefahren der Clusterförderung deutlicher $\mathrm{zu}$ machen und passgenauere Handlungsoptionen aufzuzeigen (z. B. Kiese 2008a; Kiese 2008b; Kiese 2012; Benner 2012; Häfner 2014).

Es gibt dementsprechend bereits einige Versuche, die clusterorientierte Wirtschaftsförderung systematisch zu beleuchten und $\mathrm{zu}$ verstehen. Die meisten dieser Arbeiten beschäftigen sich in erster Linie mit der Ebene der Konzeption oder der Organisation von Clusterpolitik, also allen ,staatlichen Maßnahmen zur Förderung der Entstehung und Entwicklung von Clustern“ (Kiese 2012: 77). Im Zentrum stehen dort die Akteure, die die verschiedenen Förderprogramme erstellen und deren grundsätzliche Ausrichtung festlegen oder die tatsächlichen oder anzustrebenden Inhalte der Förderprogramme selbst (z. B. Kiese 2008b; Stockinger 2010; Benner 2012; Kiese 2012; Koschatzky/ Stahlecker 2012; Ebbekink/Lagendijk 2013; Beck/Heinze/ Schmid 2014b). Im vorliegenden Beitrag steht hingegen die Ebene der praktischen Umsetzung im Zentrum, auf der die abstrakten Programme in konkrete Maßnahmen übersetzt werden. Auf dieser Ebene gibt es bisher vor allem Arbeiten, die die allgemeinen Wirkungen der Clusterförderung untersuchen (z. B. Fromhold-Eisebith/Eisebith 2008b; Wurpts/ Kirchgeorg 2013; vgl. zudem insbesondere die Evaluationen, z. B. Bührer/Kroll/Stahlecker et al. 2008; Koschatzky/Pöchhacker-Tröscher/Bührer et al. 2011) oder solche, die einzelne Aspekte herausgreifen und sich beispielsweise damit beschäftigen, wie die Clusterförderung das Gründungsverhalten (z. B. Sternberg 2014) oder die Netzwerkbildung (z. B. He/MacNeill/Wang 2014) im Cluster beeinflussen kann. Bisher fehlt es jedoch noch an Untersuchungen, die systematisch überprüfen, wie die Elemente der Clustertheorie(n) in ihrer Gesamtheit in konkrete Maßnah- men übersetzt werden und was diese Übersetzung beeinflusst. Diese Lücke will der vorliegende Beitrag schließen.

Dazu werden die zentralen Umsetzungsorganisationen der clusterorientierten Wirtschaftsförderung untersucht, die mit sämtlichen Interessenvertretern (stakeholder) der Clusterförderung und insbesondere mit den zu fördernden Unternehmen in engem Kontakt stehen und die konkreten Fördermaßnahmen entwerfen, organisieren und verwirklichen. Untersuchungsgegenstand sind die Clusterplattformen ${ }^{1}$ der „Cluster-Offensive Bayern“ (COB). Vergleichbare Organisationen werden durch die meisten deutschen Programme zur Clusterförderung eingerichtet (Ketels 2014: 49). Um die Umsetzung der Clustertheorie in die Praxis zu analysieren, werden zunächst verschiedene Clustermechanismen identifiziert, also theoretisch postulierte Zusammenhänge, Strukturen und Faktoren in Clustern, die deren Wettbewerbsfähigkeit erzeugen oder beeinflussen und die dementsprechend von den Clusterplattformen zu fördern wären (vgl. Benner 2012). Die zentrale Frage ist schließlich: Wie bearbeiten die Clusterplattformen die einzelnen Clustermechanismen und welche Faktoren beeinflussen die Förderung? Dabei wird zwischen institutionellen und strukturellen Faktoren unterschieden.

Unter strukturellen Faktoren werden Optionen oder Hindernisse verstanden, die sich aus der Position der Clusterplattformen im gesamten Fördersystem und ihrem Handlungsspielraum ergeben. Beispielsweise fehlen teilweise bestimmte Förderinstrumente oder der Zugang zu wichtigen Akteursgruppen. In diese Kategorie gehören zudem Überschneidungen der Zuständigkeiten mit anderen Förderakteuren, die einige der Clustermechanismen bereits fördern (z. B. Gründerförderung). Eine von den Clustermechanismen ausgehende systematische Analyse der strukturellen Faktoren zeigt die Schnittstellen für mögliche Kooperationen zwischen Clusterplattformen und anderen Förderakteuren, aber auch eventuelle Lücken im bisherigen Fördersystem oder bei den konkreten Förderinstrumenten.

Die institutionellen Faktoren beziehen sich hingegen auf Institutionen im soziologischen Sinn, die die Förderung bestimmter Clustermechanismen behindern oder erleichtern können. Unter Institutionen werden hier Regelsysteme verstanden, die Handlungen begrenzen, ermöglichen und formen und sie damit stabilisieren (Hasse/Krücken 2005: 13 f.). Ihre große Bedeutung im Rahmen der Clusterförde-

\footnotetext{
${ }^{1}$ Die Umsetzungsorganisationen werden im Folgenden „Clusterplattformen“ genannt, da dieser Begriff recht geläufig ist und die Umsetzungsorganisationen des hier untersuchten Förderprogramms - der „Cluster Offensive Bayern“ - so bezeichnet werden. Der Begriff „Clusterinitiative“" wird häufig synonym verwendet. Er umschließt jedoch alle organisierten Maßnahmen und bezeichnet damit nicht unbedingt nur die eigentliche Umsetzungsorganisation selbst (vgl. Lindqvist/ Ketels/Sölvell 2013: 1 f.). Der Fokus der vorliegenden Arbeit liegt hingegen eindeutig auf der Umsetzungsorganisation.
} 
Tab. 1 Interviewverzeichnis (in Reihenfolge der Durchführung)

\begin{tabular}{ll}
\hline Interviewpartner & Code für Belegstellen \\
\hline Referat für Cluster im Bayerischen Staatsministerium für Wirtschaft, Infrastruktur, Verkehr und Technologie & Ref-StMWIVT \\
Cluster Finanzdienstleistungen & CP-Fin \\
Cluster Ernährung & CP-Ern \\
Cluster Neue Werkstoffe & CP-NW \\
Cluster Nanotechnologie & CP-Nano \\
Wissenschaftlicher Beobachter 1: Prof. Dr. Matthias Kiese & WissBeo1 \\
Cluster Informations- und Kommunikationstechnik & CP-IKT \\
Cluster Bahntechnik & CP-Bahn \\
Wissenschaftlicher Beobachter 2: Prof. Dr. Knut Koschatzky & WissBeo2 \\
Referat für Cluster im Bayerischen Staatsministerium für Wirtschaft, Infrastruktur, Verkehr und Technologie & Ref-StMWIVT \\
\hline
\end{tabular}

rung ergibt sich durch die stark ausgeprägte soziale Interaktion zwischen den verschiedenen Stakeholdern (Nauwelaers 2001: 100). Die grundlegende Annahme ist, dass verschiedene Institutionen die Clusterplattformen daran hindern, die Clustermechanismen theoretisch optimal zu fördern. Den theoretischen Analyserahmen für diesen Teil bildet der Neo-Institutionalismus.

Die empirische Analyse basiert auf insgesamt zehn qualitativen leitfadengestützten Interviews mit den Geschäftsführern von sechs Clusterplattformen, dem zuständigen Betreuer der „Cluster-Offensive Bayern“ im bayerischen Wirtschaftsministerium (zwei Interviews) sowie zwei Beobachtern aus der Wissenschaft, die sich bereits mit der „ClusterOffensive Bayern“ beschäftigt hatten (vgl. Tab. 1). Die Interviews fanden 2012/2013 statt. Als Auswertungsmethode wurde die qualitative Inhaltsanalyse nach Mayring (2010) gewählt. Der Prozess der Erkenntnisgewinnung ähnelte dabei einem hermeneutischen Zirkel. Das heißt, die Erfahrungen aus den ersten Interviews sind in die folgenden Interviews eingeflossen und deren Ergebnisse wiederum dienten dazu, die bisherigen Erkenntnisse neu zu reflektieren. Darüber hinaus wurden Veröffentlichungen aller Art ausgewertet. ${ }^{2}$ Es wurden die Geschäftsführer der folgenden Clusterplattformen der „Cluster-Offensive Bayern“ interviewt: Bahntechnik, Ernährung, Finanzdienstleistungen, Informations- und Kommunikationstechnologie, Nanotechnologie und Neue Werkstoffe. Diese wurden gewählt, weil sie verschiedene Branchen repräsentieren, die sich strukturell recht stark voneinander unterscheiden. Das soll vermeiden, dass der Branchenkontext einen zu großen Einfluss auf die gewonnenen Erkenntnisse über die Arbeitsweisen der Clusterplattformen erhält.

\footnotetext{
2 Unter anderem die Webseiten der Plattformen, Broschüren der Plattformen und der Cluster-Offensive Bayern (z. B. StMWIVT 2006; StMWIVT 2012); Evaluationsberichte der Cluster-Offensive Bayern (Bührer/Kroll/Stahlecker et al. 2008; Koschatzky/PöchhackerTröscher/Bührer et al. 2011).
}

Der Beitrag gliedert sich im Weiteren wie folgt: Das nächste Kapitel stellt die „Cluster-Offensive Bayern“ und ihre Clusterplattformen sowie die Geschichte der bayerischen Förderpolitik kurz vor und beleuchtet damit den Kontext der untersuchten Akteure. Kap. 3 identifiziert die wichtigsten Erwartungsstrukturen, die andere Akteure als institutionelle Einflussfaktoren an die Clusterplattformen herantragen. Im folgenden zentralen Teil des Beitrags werden zunächst die hier untersuchten Clustermechanismen in aller Kürze vorgestellt und dann im Einzelnen daraufhin untersucht, wie die Clusterplattformen sie fördern und welche Faktoren sie dabei beeinflussen. Abschließend erfolgt eine Zusammenfassung und Diskussion der Ergebnisse.

\section{Die Cluster-Offensive Bayern}

Die „Cluster-Offensive Bayern“ ist ein bayerisches Förderprogramm, durch das im Jahr 2006 sogenannte Clusterplattformen in zunächst 19 (mit Teilclustern 21, heute nur noch 16) Themenfeldern eingerichtet wurden (vgl. Tab. 2). Die Clusterplattformen der „Cluster-Offensive Bayern“ sollen ihre jeweilige Branche landesweit fördern und unterliegen groben organisatorischen Vorgaben und Rahmenbedingungen (siehe unten). Das unterscheidet die „Cluster-Offensive Bayern“ von den meisten Programmen zur Clusterförderung anderer Bundesländer. Diese fördern tendenziell kleinräumigere und/oder thematisch enger fokussierte Clusterinitiativen (vgl. beispielhaft MfFW 2015; vgl. auch Kiese 2012). Aber auch dort werden im Allgemeinen eigens Organisationen für die Förderung gegründet oder bestehende mit ihr betraut (Ketels 2014: 49). Solange es Clusterorganisationen mit eigenem Management gibt, sollten die im Rahmen der Untersuchung der „Cluster-Offensive Bayern" gewonnenen Erkenntnisse auch übertragbar sein, da die grundsätzliche praktische Arbeit vergleichbar ist. Eine Einschränkung in der Übertragbarkeit ergibt sich nur dadurch, dass die Vielfalt der Akteure in den landesweiten und thematisch teilweise sehr breiten bayrischen Clustern 
Tab. 2 Die Clusterplattformen der „Cluster-Offensive Bayern“ (Quelle: eigene Darstellung, basierend auf StMWIVT 2012: 5)

\begin{tabular}{|c|c|}
\hline Themenfeld Mobilität & Themenfeld Mensch und Umwelt \\
\hline Automotive & Biotechnologie $^{a}$ \\
\hline Bahntechnik & Medizintechnik $^{\mathrm{a}}$ \\
\hline Logistik $^{\mathrm{a}}$ & Umwelttechnologie \\
\hline Aerospace ${ }^{b}$ & Forst und Holz \\
\hline Themenfeld Materialentwicklung & Ernährung \\
\hline Chemie & Energietechnik \\
\hline Nanotechnik & Themenfeld Informations- und Elektrotechnik \\
\hline Neue Werkstoffe & Sensorik \\
\hline Themenfeld Dienstleistungen und Medien & Leistungselektronik \\
\hline Finanzdienstleistungen & Mechatronik und Automation \\
\hline Medien $^{\mathrm{c}}$ & Informations- und Kommunikationstechnik \\
\hline
\end{tabular}

${ }^{a}$ Die Cluster waren Bestandteil der ersten Förderperiode, werden aber nicht mehr oder nur noch stark eingeschränkt im Rahmen der „ClusterOffensive Bayern“"gefördert.

${ }^{b}$ Im Cluster Aerospace wurden die Cluster Luft- und Raumfahrt sowie Satellitennavigation der ersten Förderperiode zusammengelegt.

${ }^{c}$ Der ehemalige Teilcluster Druck und Printmedien existiert weiterhin, der ehemalige Teilcluster Audiovisuelle Medien wird nicht mehr unter der „Cluster-Offensive Bayern“ gefördert.

höher als in vielen anderen ist. Dieser Umstand konfrontiert das Management unter Umständen mit etwas anderen Herausforderungen und Möglichkeiten. Zudem können die strukturellen Faktoren in einem anderen Fördersystem abweichend ausfallen.

Wie für Programme zur Clusterförderung allgemein üblich (Benneworth/Charles 2001: 394; Nauwelaers 2001: 100; Kiese 2010: 341), stellt auch die „Cluster-Offensive Bayern" keine grundsätzliche Neuausrichtung der bayerischen Förderpolitik dar, sondern ist eine kontinuierliche Weiterentwicklung vorhergehender Initiativen. Anfänge einer Politik zur Förderung von High-Tech-Branchen, Forschung und Innovationen lassen sich in Bayern bereits seit den 1970er-Jahren erkennen (Berger 2002: 11) und rückten zu Beginn der 1990er-Jahre endgültig ins Zentrum der bayerischen Wirtschaftspolitik. Durch Privatisierungen standen enorme Mittel zur Verfügung, von denen rund 4,3 Mrd. Euro (1994-2005) in das Sonderprogramm „Offensive Zukunft Bayern“ investiert wurden. Damit wurde vor allem die Forschungsinfrastruktur massiv ausgebaut, es wurden aber auch Forschungsfonds und -stiftungen sowie Organisationen zur Wirtschaftsförderung ${ }^{3}$ eingerichtet (vgl. Berger 2002). Insbesondere die letzte Phase (genannt High-Tech-Offensive; 1999-2005; 1,35 Mrd. Euro) ist ein klarer Vorläufer der „Cluster-Offensive Bayern“ (Kiese 2012: 193). Fast die Hälfte der Mittel (663,6 Mio. Euro) wurden hier zum Ausbau der Forschungsinfrastruk-

\footnotetext{
${ }^{3}$ Insbesondere wurden die staatlichen Gesellschaften „Bayern International GmbH“ (Ansiedlungsförderung) und „Bayern Innovativ“ (Innovationsförderung, Wissenstransfer) 1995 aus Mitteln der Offensive Zukunft Bayern eingerichtet.

${ }^{4}$ Life-Science, Informations- und Kommunikationstechnologie, Neue Werkstoffe, Umwelttechnik, Mechatronik.
}

tur in fünf „Hightech-Zentren von Weltrang“4 eingesetzt (Bayerische Staatskanzlei 2006: 6). Die „Cluster-Offensive Bayern“ schließt direkt daran an. Jedoch standen hier keine weiteren Privatisierungserlöse mehr zur Verfügung, sodass sich das Budget zunächst nur auf vergleichsweise geringe 45 Mio. Euro belief (2005-2011), die aus dem normalen Haushalt aufgebracht wurden. Der Schwerpunkt der Programme verschob sich daher auf die Stärkung von Innovationsprozessen in den jeweiligen Feldern.

Die Auswahl der Cluster basierte nicht auf einem Wettbewerb, sondern wurde auf der Basis von Gutachten und Expertengesprächen getroffen, was von verschiedenen Seiten, wie beispielsweise der Opposition oder den Industrie- und Handelskammern, kritisiert wurde (Kiese 2012: 201). Nach einer Evaluation der ersten Phase der „Cluster-Offensive Bayern“, die das Programm insgesamt positiv bewertete (Bührer/Kroll/Stahlecker et al. 2008; Koschatzky/Pöchhacker-Tröscher/Bührer et al. 2011), wurden einige Clusterplattformen für die zweite Förderperiode (2011-2015) umstrukturiert und teilweise ganz aus dem Programm genommen. Die Förderung wurde dann allerdings anderen Programmen oder Trägern übergeben, vollkommen eingestellt wurde keine der Plattformen. Im Rahmen der „Cluster-Offensive Bayern“ verblieben 16 Clusterplattformen.

Das übergeordnete Ziel der „Cluster-Offensive Bayern“ ist die Erhöhung der Wettbewerbsfähigkeit der bayerischen Wirtschaft, indem die Innovationsdynamik erhöht, die Produktivität gesteigert und die Bindung an den Standort verstärkt wird (StMWIVT 2006: 11; StMWIVT 2012: 6). Um diese Ziele zu erreichen, sollen Netzwerke aufgebaut und intensiviert werden. „Die Cluster-Offensive [...] ergänzt das bestehende Instrumentarium der Innovationsförderung durch das Element der Netzwerkbildung“" 
(StMWIVT 2006: 8). Diese Reduzierung auf die Förderung von Netzwerken ist für die meisten Ansätze der Clusterpolitik typisch (Raines 2000: 20; Kiese 2012: 324). Für die bayerische Regierung stehen jedoch ,nicht die Orientierung an einem theoretischen Clusterkonzept", sondern die $\mathrm{zu}$ erreichenden Ziele im Vordergrund (Bayerischer Landtag 2005: 1). Die sehr lockere Anlehnung an den Clusterbegriff kommt auch im räumlichen Zuschnitt der ausgewählten Cluster zum Ausdruck. Obwohl die meisten nur einen oder wenige räumliche Schwerpunkte haben, sind die Clusterplattformen in ihrem jeweiligen Feld für ganz Bayern zuständig. Wieder andere bilden überhaupt keine deutlichen Agglomerationen aus, was den Clusterbegriff ebenso unterminiert (vgl. Bayerischer Industrie- und Handelskammertag 2006; Kiese 2012: 203 f.).

Alle Plattformen haben einen recht ähnlichen organisatorischen Aufbau (vgl. Bührer/Kroll/Stahlecker et al. 2008). Sie haben einen sogenannten Clustergeschäftsführer und ein unterschiedlich großes Team, welches das operative Geschäft ausführt. Zudem haben alle einen oder mehrere ehrenamtliche Clustersprecher benannt, die den Cluster repräsentieren und als ,Türöffner" dienen sollen. Dabei handelt es sich um im jeweiligen Feld angesehene Persönlichkeiten, wie Unternehmer und Professoren. Über diese Grundstruktur hinaus haben die meisten Plattformen Gremien eingerichtet, die die Plattformen strategisch beraten, teilweise Entscheidungen treffen und die kurz- und mittelfristigen Aktionen bestimmen. Die formale Organisationsform unterscheidet sich von Cluster zu Cluster. Viele sind als Verein organisiert, einige aber auch als $(\mathrm{g}) \mathrm{GmbH}$, Projektabteilung oder Geschäftsstelle innerhalb einer größeren Organisation. Der operative Sitz der Plattformen wurde in den meisten Fällen bei bereits bestehenden Organisationen angesiedelt, die sich schon vorher mit dem jeweiligen Thema auseinandergesetzt haben.

Neben der degressiven Grundfinanzierung durch das bayerische Wirtschaftsministerium sollen sich die Clusterplattformen auch selbst finanzieren. Bis 2015 soll sich der Eigenanteil auf mindestens 50 Prozent belaufen und zukünftig weiter gesteigert werden. Die wichtigsten Finanzierungsquellen sind Mitgliedsbeiträge (bei Vereinen) und Veranstaltungsbeiträge. Hinzu kommen kostenpflichtige Dienstleistungen wie Auftragsstudien, Anzeigen in Newslettern, Beratungsleistungen und Gebühren für Messebeteiligungen. Ferner sollen die Plattformen die bayerische Wirtschaft dabei unterstützen, weitere Fördergelder von Land, Bund oder EU einzuwerben. Ein Teil dieser Mittel kommt auch den Clusterplattformen direkt zu Gute, falls diese beispielsweise das Projektmanagement übernehmen.

Die Maßnahmen aller Clusterplattformen sind im Kern unterschiedlich große Veranstaltungen und Projekte. Hierbei gibt es idealtypisch eine Schrittfolge, die von großen über kleinere Veranstaltungen zu konkreten Projekten führt
(CP-NW). Nahezu alle Plattformen veranstalten jährlich einen großen Kongress, der als eine Art Leitveranstaltung mit einem mehr oder weniger breiten Themenangebot viele Besucher anlocken soll. Hier gibt es im Allgemeinen Vorträge zu verschiedenen Themen und - aufbauend auf dem Feedback - werden weitere Veranstaltungen organisiert, die einzelne Themen vertiefen. Beliebt sind dafür sogenannte Clustertreffs, die in den Örtlichkeiten von Unternehmen oder Forschungsinstituten stattfinden. Generiert ein Thema dauerhaftes Interesse, werden häufig Arbeitskreise eingerichtet, in denen sich die entsprechenden Experten regelmäßig austauschen. Der ,Traum` der Clustermanager sind die Projekte (CP-NW). In diesen beteiligen sich mehrere Partner zum Beispiel an der konkreten Markteinführung eines Produkts oder einer Technologie. Die Clusterplattformen sind dabei meist nur an der Vorbereitung und Beratung sowie gelegentlich am Projektmanagement beteiligt. In den Projekten sehen die Plattformen eine erfolgreiche Vernetzung und damit ihr Ziel erreicht (CP-Bahn, CP-NW).

Neben den Veranstaltungen und Projekten bieten die Plattformen weitere Maßnahmen an. Das Angebot umfasst unter anderem die Vermittlung von Ansprechpartnern, Beratungen, Internetplattformen und Newsletter, Maßnahmen zur Aus- und Weiterbildung, Öffentlichkeitsarbeit, Weiterentwicklung von Studiengängen, Vermittlung von Förderprogrammen oder Gemeinschaftsstände auf Messen. Dem Evaluationsbericht zu Folge werden die Angebote in unterschiedlichem Umfang genutzt. Während die Veranstaltungen und Newsletter von zwei Dritteln der erreichten Akteure zumindest punktuell genutzt werden, werden andere Angebote deutlich weniger in Anspruch genommen (Koschatzky/Pöchhacker-Tröscher/Bührer et al. 2011: 24).

\section{Interessen und Erwartungsstrukturen im organisationalen Feld der Clusterplattformen}

Die Interessen und Erwartungen, die andere Akteure an die Clusterplattformen richten, bilden die institutionellen Einflussfaktoren auf deren Arbeitsweise. Um diesen $\mathrm{Zu}$ sammenhang theoretisch $\mathrm{zu}$ fassen, greift die vorliegende Untersuchung auf den Neo-Institutionalismus zurück. Die Grundannahme dieses organisationssoziologischen Ansatzes ist, dass institutionalisierte Erwartungsstrukturen in einem organisationalen Feld die innere Struktur und die Handlungen einer Organisation bestimmen (Meyer/Rowan 1977; DiMaggio/Powell 1983; Scott 2001: 51). Das organisationale Feld konstituiert sich aus den Akteuren, die für eine Organisation relevant sind. Die Erwartungen dieser Akteure wirken als sozial verbindliche Institutionen auf die Organisation ein und prägen sie in hohem Maße. Handlungen sind im Neo-Institutionalismus dementsprechend kein Ergebnis von unabhängigen, situativen und rationa- 
len Entscheidungen einzelner Akteure, sondern umfassend strukturiert durch die Erwartungen anderer Akteure (Hasse/ Krücken 2005: 64). Eine zentrale Rolle nimmt dabei das Konzept der Legitimität ein. Es wird angenommen, dass der Legitimitätsgewinn das oberste Ziel einer Organisation ist, da sie sonst nicht in der Lage ist, benötigte Ressourcen oder Kooperationspartner zu mobilisieren (Hellmann 2006: 75). Legitimität, also Anerkennung, Vertrauen oder Integrität, wird einer Organisation zugeschrieben, wenn sie den Erwartungen in ihrem organisationalen Feld entspricht (Walgenbach/Meyer 2008: 64). Die Konsequenz aus dem Streben nach Legitimität ist, dass technisch rationale Effizienz und sachgerechte Zielverfolgung in der Aktivität der Organisationen in den Hintergrund treten und stattdessen Handlungen priorisiert werden, die eine Anpassung an die Erwartungsstrukturen im organisationalen Feld demonstrieren (Meyer/Rowan 1977; Tacke 2006: 90). Übertragen auf die hier untersuchten Akteure bedeutet das, dass sie keine theoretisch optimale Clusterförderung betreiben können, da der zu erwartende Legitimitätsgewinn oder -verlust bei Entscheidungen für oder gegen eine Maßnahme eine wichtige Rolle spielt.

Die empirische Untersuchung zeigt, dass die Aktivität der Clusterplattformen und ihre internen Strukturen, wie vom Neo-Institutionalismus postuliert, in der Tat sehr stark darauf ausgerichtet sind, Legitimität zu erlangen (vgl. Häfner 2014: 121). Der Grund dafür sind die ausgeprägten Abhängigkeitsverhältnisse, in denen die Plattformen stehen. Einerseits unterliegen sie den Vorstellungen und Erwartungen der Politik, die sie eingerichtet hat und gegebenenfalls auch wieder einstellen kann, andererseits müssen sie den Erwartungen der geförderten Akteure entsprechen. Die Abhängigkeiten ergeben sich aus der partizipativen Funktionsweise der Clusterförderung (vgl. Nauwelaers 2001: 100; Raines 2001). Diese soll soziale Prozesse zwischen den regionalen Wirtschaftsakteuren anregen und erfordert damit eine aktive Beteiligung der Clusterplattformen an den wirtschaftlichen Maßnahmen. Dazu müssen die Plattformen jedoch als ,Mitspieler' anerkannt werden, was sie nur erreichen können, indem sie die ,Spielregeln ' befolgen und ihre Relevanz für die geförderten Akteure beweisen. Die Notwendigkeit, sich selbst zu finanzieren, verstärkt die Abhängigkeiten zusätzlich. Insgesamt ergibt sich ein hoher Rechtfertigungsdruck für die Clusterplattformen, dem sie nur standhalten können, indem sie sich und ihre Maßnahmen an die verschiedenen Erwartungen anpassen.

Welche Erwartungen und Interessen der Politik und der geförderten Akteure lassen sich also identifizieren? Die Politik, hier vertreten durch das bayerische Wirtschaftsministerium, fordert von den Clusterplattformen zunächst einige allgemeine Dinge. Beispielsweise wird erwartet, dass sie möglichst viele Clustermitglieder erreichen, dass sie ihren Eigenanteil an den Finanzmitteln erhöhen oder dass sie da- zu beitragen, weitere Fördergelder einzuwerben. Das Ministerium überprüft laufend, ob diese Ziele erreicht werden und hat zudem tiefergehende Evaluationen in Auftrag gegeben (Ref-StMWIVT). Bei der inhaltlichen Ausrichtung werden die Plattformen ,an der langen Leine“ (RefStMWIVT, WissBeo2) gelassen. Es ist jedoch zu erkennen, dass der Fokus auf die Förderung von Netzwerken bereits durch die Politik angelegt wurde. Maßnahmen zum Aufbau und zur Intensivierung von Netzwerken sind daher sehr präsent (CP-NW).

Aufgrund politischer Vorgaben ist die Clusterförderung zudem auf bayerische Akteure fokussiert. Das bedeutet allerdings nur, dass der Großteil der durch die Förderung zu erwartenden Wertschöpfung in Bayern stattfinden soll und schließt die Beteiligung von nicht-bayerischen Akteuren keineswegs aus (CP-Fin, CP-NW). Die einzigen konkreten Maßnahmen, die die Politik von den Plattformen einfordert, sind solche zum Standortmarketing (Ref-StMWIVT). Das betrifft vor allem internationale Messen oder ähnliches, auf denen die Clusterplattformen explizit als Vertreter der bayerischen Wirtschaft Präsenz zeigen sollen.

Darüber hinaus beeinflussen politische Leitlinien für die einzelnen Branchen die Unternehmungen der Plattformen, weswegen sie sich intensiv mit den jeweiligen Fachreferaten in den Ministerien abstimmen. Inwiefern diese Tatsache den Umgang der Plattformen mit einzelnen Clustermechanismen beeinflusst, hängt jedoch vom konkreten Inhalt der politischen Leitlinien $a b$ und kann nicht verallgemeinert werden. Dieser Kommunikationskanal zwischen Ministerien und Clusterplattformen wird zudem genutzt, um Informationen über Entwicklungen und eventuelle Problemlagen in der Wirtschaft zu gewinnen (Ref-StMWIVT).

Seitens der Akteure, an die sich die Förderung richtet - in erster Linie Unternehmen, aber beispielsweise auch Forschungseinrichtungen -, werden vor allem drei institutionalisierte Erwartungsstrukturen deutlich. Erstens wird ein direkter, möglichst kurzfristiger und kalkulierbarer oder zumindest abschätzbarer Nutzen von den Aktionen der Plattformen erwartet. Zweitens wollen die Unternehmen ihr Wissen geschützt wissen und drittens sollen sich die Plattformen neutral verhalten. Daraus leiten sich einige Verhaltensweisen der Plattformen ab, die ihre Handlungsspielräume in hohem Maße bestimmen.

Die Erwartung eines direkten Nutzens durch die Fördermaßnahmen bewirkt, dass sich die Clusterplattformen sehr stark an konkret geäußerten Bedürfnissen der geförderten Akteure orientieren müssen. Der Nutzen jeder Maßnahme muss prägnant und schnell erkennbar sein, um Hemmschwellen zur Teilnahme zu senken (CP-Ern, CP-NW). Die Maßnahmen (insbesondere die Projekte) haben daher im Allgemeinen sehr konkrete und fokussierte Inhalte, die eine eng eingegrenzte Akteursgruppe interessieren und beispielsweise eine konkrete Technologie oder ein bestimmtes 
Produkt in den Mittelpunkt stellen. Die Förderung von Clustermechanismen, die einen eher langfristigen Fokus haben (z. B. Aufbau von globalen Pipelines), deren Nutzen nur schwer kalkulierbar ist (z.B. Kooperation zwischen Wissenschaft und Wirtschaft) oder die konkreten Interessen widersprechen können (z. B. intensiver Wettbewerb), tritt als Konsequenz in den Hintergrund. Es darf dennoch nicht außer Acht gelassen werden, dass eine langfristige Clusterentwicklung nur über kleine Schritte funktionieren kann.

Die Erwartung der Unternehmen, dass ihr Wissen geschützt werden muss und nicht an andere Akteure,verloren “ gehen darf, äußert sich in einer gewissen Verschlossenheit gegenüber den Clusterplattformen und deren Maßnahmen (CP-NW). Da die Intensivierung von Wissenstransfers aber ein zentrales Element der Clusterförderung ist, kann es hier zu Konflikten kommen. Die Untersuchung hat jedoch gezeigt, dass dieses Problem nicht überbewertet werden sollte (Ref-StMWIVT). Nach Ansicht der Clustermanager sind die Unternehmen den Plattformen und ihren Maßnahmen gegenüber im Allgemeinen recht offen und wenn sie für sich einen klaren Nutzen erkennen können, sind sie auch bereit, ihr Wissen zu teilen. Entscheidend ist die Fähigkeit der Clustermanager, bei den geförderten Akteuren Vertrauen aufzubauen. Sie benötigen daher eine hohe Sozialkompetenz (vgl. Fromhold-Eisebith/Eisebith 2008a: 87; JappeHeinze/Baier/Kroll 2008: 10 f.) und müssen durch persönlichen Kontakt und klärende Gespräche Überzeugungsarbeit leisten. Als vorteilhaft hat sich zudem die Einbindung von renommierten Persönlichkeiten in die Projekte und die Strukturen der Clusterplattformen erwiesen. Diese können somit ihre eigenen Netzwerke einbringen und als ,Türöffner' wirken. Falls die beteiligten Unternehmen bei einer Maßnahme dennoch befürchten, wettbewerbsrelevantes Wissen an Konkurrenten zu verlieren, werden in seltenen Fällen auch einzelne Akteure von Maßnahmen ausgeschlossen. Nach Aussage aller Interviewpartner kann die Verschlossenheit der Unternehmen trotz allem in einigen Fällen eine echte Grenze für die Handlungsmöglichkeiten der Plattformen darstellen.

Die Clustermanager legen sehr viel Wert auf die Neutralität der Plattformen gegenüber allen beteiligten Akteuren. Diese beschreiben sie als essenziell und gleichzeitig auch als ihre größte Stärke im Vergleich zu anderen Förderakteuren. Neutralität bedeutet hier, dass sie keine einzelnen Unternehmen oder bestimmte Teilbereiche bevorzugen und auch nicht als Interessenvertreter der Politik auftreten. Die Unternehmen loben die Plattformen teilweise explizit als einen Raum, in dem auf neutralem Boden Möglichkeiten und Bedingungen für Kooperationen vorab abgeklärt werden können (CP-Bahn). Die Neutralität wird von den Clustermanagern bewusst verteidigt und sie nehmen daher gegebenenfalls Abstand von Maßnahmen, die eine Positionierung erfordern würden.

Neben diesen drei grundsätzlichen Erwartungen - direkter Nutzen, Schutz des Wissens, Neutralität - müssen sich die Plattformen ferner an branchenspezifische Normen anpassen. Beispielsweise ist beim Cluster Finanzdienstleistungen das Prestige der Sprecher auf Veranstaltungen von großer Bedeutung, während beim Cluster Informationsund Kommunikationstechnik wenig Wert auf Eigenmarketing gelegt wird. Derartige Anpassungen lassen sich jedoch kaum verallgemeinern und wirken sich zudem eher auf die Herangehensweise an bestimmte Tätigkeiten aus, als auf deren Ziele.

\section{Umgang mit Clustermechanismen durch die Clusterplattformen}

\subsection{Zusammenstellung der Clustermechanismen}

Eine umfassende Clustertheorie existiert nicht und ist auch nicht absehbar (vgl. Kiese 2008a: 49). Die grundlegende Feststellung, dass sich ökonomische Aktivität und einzelne Branchen im Raum konzentrieren, ist ein zentraler Forschungsgegenstand der Wirtschaftsgeographie, weswegen die theoretischen Arbeiten dazu sehr vielfältig und umfassend sind. Für die vorliegende Untersuchung wurde daher eine eklektische Sammlung von Mechanismen zusammengestellt, die auf verschiedenen Beiträgen zur Clustertheorie basiert. Diese Sammlung soll weniger theoretisch umfassend und stringent sein, sondern vor allem die Vielzahl möglicher Ansatzpunkte für die Clusterförderung aufzeigen. Die Zusammenhänge zwischen den einzelnen Clustermechanismen treten dabei notwendigerweise in den Hintergrund. Da diese jedoch von Cluster zu Cluster unterschiedlich wirken, wird dieser Schwachpunkt einer eklektischen Zusammenstellung, die einen allgemeingültigen Überblick verschaffen soll, an dieser Stelle akzeptiert. Es sei dennoch mit Nachdruck darauf hingewiesen, dass in einem gegebenen Cluster nicht einfach nur alle Mechanismen möglichst umfassend zu fördern sind. Vielmehr kommt es im Einzelfall stark darauf an, das Wechselspiel zwischen den Mechanismen zu beachten und $\mathrm{zu}$ analysieren, um daraufhin die jeweilige Förderstrategie zu entwickeln. Die einzelnen Clustermechanismen und ihre Wirkungsweise können an dieser Stelle nicht umfassend erklärt werden. Dafür sei auf die jeweils angegebene Literatur verwiesen (vgl. Tab. 3).

Der erste Block der aufgeführten Mechanismen betrifft soziale Interaktionen, deren Förderung im Allgemeinen die Kernaufgabe der Clusterplattformen ist. Bereits mehrfach haben Wissenschaftler festgestellt (und kritisiert), dass Clusterförderung meist auf den Aufbau von Netzwerken reduziert wird (vgl. z. B. Raines 2000: 20; Kiese 
Tab. 3 Funktionsweise der Clustermechanismen (Quelle: eigene Darstellung nach Häfner 2014: 29 ff.)

\begin{tabular}{|c|c|c|}
\hline Clustermechanismus & Funktionsweise & Autoren (Auswahl) \\
\hline \multicolumn{3}{|l|}{ Interaktion } \\
\hline \multicolumn{3}{|l|}{ Nach Art der Interaktion } \\
\hline Stand-by-Kontakte & Stand-by-Kontakte sind eine Vorstufe für weitere Kooperationen & - \\
\hline $\begin{array}{l}\text { Rauschen, Cafeteria- } \\
\text { Effekte }\end{array}$ & $\begin{array}{l}\text { Ungezielte, ungeplante Zirkulation von Wissen, Informationen, Neuigkeiten, Gerüch- } \\
\text { ten ermöglicht den Teilnehmern, Chancen, Potenziale, Gefahren oder Tätigkeiten von } \\
\text { sich selbst, anderen, dem Markt, derTechnologie usw. zu erkennen und einzuschätzen }\end{array}$ & $\begin{array}{l}\text { Camagni (1991); Grabher } \\
\text { (2002); Bathelt/Malmberg/ } \\
\text { Maskell (2004); Storper/ } \\
\text { Venables (2004) }\end{array}$ \\
\hline $\begin{array}{l}\text { Communities of Prac- } \\
\text { tice }\end{array}$ & $\begin{array}{l}\text { Expertenkreise sind eine hoch spezialisierte und sehr effektive und innovative Sonder- } \\
\text { form der sozialen Netzwerke. Sie sind zielgerichteter und damit effektiver, aber auch } \\
\text { beschränkter als lediglich eine Teilnahme am Rauschen }\end{array}$ & $\begin{array}{l}\text { Lissoni (2001); Gertler } \\
\text { (2003) }\end{array}$ \\
\hline Konkrete Kooperation & Direkte Kooperationen führen zu intensivem und zielgerichtetem Wissensaustausch & $\begin{array}{l}\text { Malmberg/Maskell (2002); } \\
\text { Grabher (2002) }\end{array}$ \\
\hline \multicolumn{3}{|l|}{ Nach Interaktionspartner } \\
\hline Vertikal - horizontal & $\begin{array}{l}\text { Interaktionen entlang vertikaler Beziehungen ermöglichen gemeinsames Lernen und } \\
\text { den Austausch von implizitem Wissen. Die Wissenspools und Kompetenzen sind hier } \\
\text { relativ heterogen und potenzielle Gewinne daher recht groß. Interaktionen entlang } \\
\text { horizontaler Beziehungen ermöglichen Beobachtung und Vergleich. Unternehmen } \\
\text { können aus Fehlern und Erfolgen von Konkurrenten lernen und dieses Wissen in die } \\
\text { eigene Arbeit einfließen lassen. Zwischen beiden Dimensionen besteht ein Ausgleich } \\
\text { (trade-off) }\end{array}$ & $\begin{array}{l}\text { Camagni (1991); Glaeser/ } \\
\text { Kallal/Scheinkman et al. } \\
\text { (1992); Porter (1998a); } \\
\text { Maskell/Malmberg (1999); } \\
\text { Malmberg/Maskell (2002); } \\
\text { Malmberg/Power (2005); } \\
\text { Bathelt/Glückler (2012) }\end{array}$ \\
\hline Intern - extern & $\begin{array}{l}\text { Externe Kooperationen bringen neues Wissen in den Cluster. Zu starke interne Bin- } \\
\text { dungen führen zu Vertrauensseligkeit und erhöhen die Gefahr eine Sperrung (lock-in). } \\
\text { Zudem können sie ausschließend wirken. Schwache interne Bindungen verhindern } \\
\text { Austauscheffekte (spillover) }\end{array}$ & $\begin{array}{l}\text { Maillat (1998); Scott } \\
\text { (1998); Bresnahan/ } \\
\text { Gambardella/Saxenian } \\
\text { (2001); Bathelt/Malmberg/ } \\
\text { Maskell (2004); Bathelt/ } \\
\text { Glückler (2012) }\end{array}$ \\
\hline $\begin{array}{l}\text { Wissenschaft - Wirt- } \\
\text { schaft }\end{array}$ & $\begin{array}{l}\text { Ergebnisse aus Forschungseinrichtungen können schnell ökonomisch in Wert gesetzt } \\
\text { werden. Wissenschaftliche Forschungen können an die Bedarfe der Wirtschaft ange- } \\
\text { passt werden }\end{array}$ & $\begin{array}{l}\text { Porter (1998a); Bathelt/ } \\
\text { Malmberg/Maskell (2004) }\end{array}$ \\
\hline
\end{tabular}

2012: 324). Clusterförderung ist in der Praxis zwar äußerst vielfältig, aber im Kern geht es so gut wie immer um Interaktionen und Netzwerke, die Wissenstransfers zwischen verschiedenen Akteuren ermöglichen oder erleichtern sollen. Die „Cluster-Offensive Bayern“ ist an dieser Stelle keine Ausnahme. Auch die Clustertheorien betonen die herausragende Bedeutung von sozialen Interaktionen für Cluster. So schreibt Porter (1998a: 225): ,Social glue binds clusters together." Interaktionen sind in den Clustertheorien jedoch nie das einzige Element.

Neben verschiedenen Mechanismen, die Formen von Interaktionen sind oder sie befördern können, findet sich in der Zusammenstellung eine Betrachtung der Interaktionen nach Interaktionspartnern. Diese ist ein Versuch, zumindest für diesen zentralen Bereich den Umgang der Plattformen mit dem Zusammenspiel der Clustermechanismen zu beachten. Hier geht es um die Frage, wie die Clusterplattformen die Balance zwischen der vertikalen und der horizontalen bzw. der internen und der externen Clusterdimension finden (vgl. Bathelt/Glückler 2012: 260 ff.). Zudem wird hier die Förderung der Interaktion zwischen Wissenschaft und Wirtschaft gesondert betrachtet, die insbesondere die Politik als sehr wichtig ansieht (Boekholt/Thuriaux 1999: 405 f.; Raines 2001: 7). Insgesamt können die Clusterplatt- formen alle Mechanismen, die die Interaktion betreffen, recht gut fördern, was auch nicht weiter verwunderlich ist, da sie dazu eingerichtet wurden. Gäbe es hier grundlegende Probleme, wären sie schon lange eingestellt oder umstrukturiert worden. Eine Ausnahme ist die Einbindung externer Partner, die aus strukturellen Gründen sehr schwierig ist.

Den zweiten Block bilden Mechanismen, die nicht auf direkte soziale Interaktion abzielen. Hierzu zählen vor allem die verschiedenen Faktorinputs, aber auch Mechanismen wie der Wettbewerb oder die Nachfragebedingungen, die z. B. Porter (1998a: 211 f.; Porter 1998b) stark betont. In diesen Handlungsfeldern sind im bayerischen Kontext bereits viele andere Förderakteure aktiv, mit denen die Clusterplattformen verstärkt kooperieren (müssen) oder an die sie weitervermitteln. Die Arbeitsteilung ergibt sich zum Teil dadurch, dass hier Förderinstrumente benötigt werden, die den Clusterplattformen nicht zur Verfügung stehen (z.B. Beteiligungskapital zur Gründerförderung).

\subsection{Umgang mit den Clustermechanismen}

Die Bearbeitung der einzelnen Clustermechanismen durch die Clusterplattformen stellt sich wie folgt dar: 
Tab. 3 Funktionsweise der Clustermechanismen (Quelle: eigene Darstellung nach Häfner 2014: 29 ff.) (Fortsetzung)

\begin{tabular}{|c|c|c|}
\hline Clustermechanismus & Funktionsweise & Autoren (Auswahl) \\
\hline \multicolumn{3}{|l|}{ Weitere Faktoren } \\
\hline \multicolumn{3}{|l|}{ Arbeitsmarkt } \\
\hline Quantitativ & $\begin{array}{l}\text { Ein großer spezialisierter Arbeitsmarkt ermöglicht einfaches Finden von Arbeitneh- } \\
\text { mern bzw. Arbeitgebern }\end{array}$ & \multirow{3}{*}{$\begin{array}{l}\text { Camagni (1991); Krugman } \\
\text { (1991); Porter (1998a); } \\
\text { Power/Lundmark (2004); } \\
\text { Malmberg/Power (2005); } \\
\text { Glückler (2005) }\end{array}$} \\
\hline Qualitativ & Eine hohe Qualifikation der Arbeitnehmer ermöglicht hohe Innovationsraten & \\
\hline Mobilität & $\begin{array}{l}\text { Die Mobilität der Arbeitnehmer ermöglicht Wissensaustausch und soziale Bindungen } \\
\text { zwischen verschiedenen Unternehmen }\end{array}$ & \\
\hline \multicolumn{3}{|l|}{ Neue Unternehmen } \\
\hline Externe & Unternehmen von außen bringen neues Wissen und neue Kompetenzen in den Cluster & \multirow{2}{*}{$\begin{array}{l}\text { Porter (1998a); Feldman/ } \\
\text { Francis/Bercovitz (2005); } \\
\text { Boschma/Wenting (2007); } \\
\text { Dahl/Østergaard/Dalum } \\
\text { (2010) }\end{array}$} \\
\hline Start-ups/Spinn-offs & $\begin{array}{l}\text { Neugründungen von Unternehmen bringen neues Wissen und neue Kompetenzen in } \\
\text { den Cluster und ,testen“ Innovationen. Spinn-offs haben häufig enge soziale Verbin- } \\
\text { dungen zu den Ursprungsunternehmen und ermöglichen so einen intensiven Wissens- } \\
\text { austausch }\end{array}$ & \\
\hline Wettbewerb/Rivalität & $\begin{array}{l}\text { Intensiver ökonomischer oder sozialer Wettbewerb führt zu Innovationsdruck und } \\
\text { damit hohen Innovationsraten }\end{array}$ & $\begin{array}{l}\text { Porter (1998a), (1998b); } \\
\text { Malmberg/Power (2005) }\end{array}$ \\
\hline $\begin{array}{l}\text { Spezialisierte Infra- } \\
\text { struktur }\end{array}$ & $\begin{array}{l}\text { Spezialisierte Infrastrukturen wie z. B. Forschungsinstitute oder Bildungseinrichtun- } \\
\text { gen sind ein wichtiger Standortvorteil }\end{array}$ & Porter (1998a) \\
\hline $\begin{array}{l}\text { Institutionelle Rah- } \\
\text { menbedingungen }\end{array}$ & Beeinflussen soziale und ökonomische Interaktionen & $\begin{array}{l}\text { Porter (1998a); Glückler } \\
\text { (2005); Gertler (2010) }\end{array}$ \\
\hline Formell & Festgeschriebene Regeln, Gesetze & \\
\hline Informell & z. B. Werte, Normen, Traditionen & \\
\hline Zugang zu Kapital & $\begin{array}{l}\text { Der Zugang zu (Risiko-)Kapital erleichtert die Einführung und Etablierung von neuen } \\
\text { Innovationen }\end{array}$ & Ferrary/Granovetter (2009) \\
\hline Bekanntheit/Image & $\begin{array}{l}\text { Bekanntheit und positives Image eines Clusters locken externe Unternehmen, Arbeit- } \\
\text { nehmer und Investitionen an }\end{array}$ & Porter (1998a) \\
\hline Nachfragebedingungen & Spezialisierte, anspruchsvolle Nachfrage regt Innovationen an & Porter (1998a) \\
\hline
\end{tabular}

Vernetzungsoptionen. Die Clusterplattformen sind sehr gut in der Lage, Vernetzungsoptionen für die von ihnen geförderten Akteure zu schaffen. Da sie sehr viele Unternehmen und Einrichtungen im Cluster kennen, ist es kein Problem, verschiedene Akteure miteinander gezielt bekannt zu machen. Teilweise existieren dafür Kompetenzdatenbanken. Daneben sind vor allem die Veranstaltungen sehr gut geeignet, um Vernetzungsoptionen zu schaffen. Da die Plattformen hier im Interesse aller handeln, gibt es dabei keine Probleme. Ein kleineres strukturelles Problem besteht darin, den Überblick zu behalten (CP-Ern, CP-IKT).

Rauschen. Die Förderung des lokalen ,Rauschen ' gelingt den Clusterplattformen ebenso gut. Insbesondere ihre Veranstaltungen bieten viele Gelegenheiten für den ungezwungenen Austausch zwischen den Teilnehmern. Dem wird im Rahmenprogramm auch gezielt Raum gegeben und die Plattformen werden von den Unternehmen teilweise explizit für diese Möglichkeiten gelobt (CP-Fin). Daneben geben die Plattformen durch Newsletter und Internetplattformen weiteren Input ins lokale Rauschen.

Communities of Practice. Die Clusterplattformen richten häufig Arbeitskreise, aber auch Beiräte oder ähnliche Gremien ein. Das machen sie, da sie darin bereits eine er- folgreiche Vernetzung sehen, vor allem aber benötigen sie diese Kreise ganz grundsätzlich für ihre Arbeit. Viele Ideen für Maßnahmen, aber auch die allgemeine Strategie werden dort generiert (CP-IKT). Da die Mitglieder dieser Kreise im Allgemeinen anerkannte Experten im jeweiligen Feld sind, bauen die Plattformen somit gezielt lokale communities of practice auf. Verbindungen zu globalen communities of practice sind für einen Cluster sicherlich noch wertvoller. Auch zu diesen können die Clusterplattformen einen guten Zugang bekommen, wenn es ihnen gelingt, deren Mitglieder in ihre Strukturen einzubinden. Da sich Vertreter von bayerischen Großkonzernen und Wissenschaftler häufig in den Plattformen engagieren, haben die bayerischen Plattformen Zugang zu globalen communities of practice und können diese für sich nutzen.

Konkrete Kooperationen. Der Aufbau von konkreten Kooperationen zwischen verschiedenen Clusterakteuren hat innerhalb der Aktivität der Clusterplattformen eine sehr hohe Priorität - teilweise wird er sogar als zentrales Ziel beschrieben - und wird insbesondere durch die Projekte etabliert (CP-NW). Die Arbeit mit Projekten bietet für die Plattformen einige Vorteile. Die Einrichtung eines Projektes ist zunächst eine sichtbare Vernetzung und damit ein erfolgreicher Schritt zur Clusterentwicklung. Zusätzlich gibt 
es viele praktische Anreize für die Plattformen, Projekte zu initiieren. Diese sind zunächst gegenüber der Politik sehr gut nachweisbar, was beispielsweise bei der Förderung des Rauschens oder von Vernetzungsoptionen nicht direkt der Fall ist (CP-Nano). Zudem sind Projekte eine wichtige Geldquelle für die Clusterplattformen, was im Hinblick auf den geforderten Eigenanteil sehr wichtig ist. Das Projektmanagement können sich die Plattformen bezahlen lassen und zudem können für Projekte weitere Fördergelder aus verschiedenen Quellen eingenommen werden. Gegenüber den geförderten Akteuren bieten Projekte den Vorteil, dass die Inhalte klar benannt werden können und der Nutzen meist recht gut kalkulierbar ist, was die Hemmschwellen zum Engagement senkt (CP-NW). Zudem gibt es gerade bei kleinen und mittleren Unternehmen (KMU) häufig einen echten Bedarf an Unterstützung. Die Angst der Unternehmen, ihr Wissen an andere zu verlieren, kann jedoch ein Problem bei der Förderung von konkreten Kooperationen werden. Daher ist das Projektmanagement teilweise recht aufwendig, und unter Umständen wird die Kooperation auch verhindert. Kritisch ist anzumerken, dass die Projekte meist mit nur wenigen Clusterakteuren durchgeführt werden.

Interaktionen in der vertikalen bzw. der horizontalen Clusterdimension. Interaktionen entlang von vertikalen Produktionsketten können durch die Clusterplattformen vergleichsweise gut gefördert werden. Insbesondere die technologisch orientierten Cluster versuchen meist gezielt, „Wertschöpfungsketten zu schließen“ (CP-NW). Die Förderung funktioniert hier recht problemlos, da die Unternehmen häufig einen hohen Bedarf an Unterstützung haben (CP-Bahn). Die Einführung neuer Technologien in Produktionsnetze benötigt immer eine intensive Koordination zwischen den Unternehmen, die insbesondere kleine und mittlere Unternehmen vor große Herausforderungen stellt (CP-NW). Der Nutzen von Kooperationen auf vertikaler Ebene ist zudem kalkulierbar und im Normalfall haben alle Beteiligten ein gemeinsames Interesse am Erfolg. Darüber hinaus ist die Gefahr geringer, Wissen an Konkurrenten zu verlieren, und Trittbrettfahrereffekte spielen kaum eine Rolle. Auch Interaktionen zwischen Clusterakteuren auf der horizontalen Ebene, also zwischen potenziellen Konkurrenten, können gut durch die Plattformen gefördert werden, solange gemeinsame Interessen verfolgt werden. Typisch sind hier beispielsweise gemeinsame Maßnahmen zur Absatzförderung (CP-IKT). Derartige Maßnahmen laufen meist problemlos ab, da der Koordinationsbedarf relativ gering ist und selten komplexes Wissen im Zentrum steht. Sobald die Interessen allerdings gegenläufig sind oder die Gefahr besteht, Wissen an Konkurrenten zu verlieren, können die Plattformen keine Maßnahmen mehr durchführen. Der zentrale Nutzen einer ausgeprägten horizontalen Clus- terdimension, der vereinfachte Vergleich mit Konkurrenten (vgl. Malmberg/Maskell 2002), wird damit nur zum Teil aktiviert.

Eine eindeutige Bevorzugung von Fördermaßnahmen entlang der vertikalen oder der horizontalen Clusterdimension konnte nicht festgestellt werden. Grundsätzlich orientieren sich die Plattformen stark an den vorhandenen Strukturen im Cluster. Ist die vertikale Dimension bedeutender (z. B. Cluster Neue Werkstoffe), werden Fördermaßnahmen entlang von Produktionsketten deutlich bevorzugt. Spielen vertikale Zusammenhänge hingegen eine untergeordnete Rolle (z. B. Cluster Finanzdienstleistungen, Cluster Informations- und Kommunikationstechnik), sind Maßnahmen mit Akteuren, die auf der gleichen Wertschöpfungsstufe stehen, der Normalfall. Die Ursache dafür ist die ausgeprägte Orientierung an konkreten Bedürfnissen.

\section{Interaktionen zwischen internen bzw. mit externen Ak-} teuren. Die Aufgabe, lokale Akteure mit externen zu verbinden, stellt die Clusterplattformen vor größere Schwierigkeiten, obwohl sich die Clustermanager dieses Problems bewusst sind und sie daran arbeiten (CP-Bahn, CP-Nano, CP-IKT). Die zentralen Schwierigkeiten sind struktureller Natur. Den Clusterplattformen fehlen der Überblick über potenziell interessante externe Akteure und vor allem der Zugang zu diesen. Für Bayern kommt hinzu, dass die internationale Vernetzung der bayerischen Wirtschaft die Aufgabe eines anderen Förderakteurs (Bayern International) ist, mit dem die Plattformen allerdings gegebenenfalls zusammenarbeiten. Um Interaktionen mit externen Akteuren dennoch zu fördern, kooperieren die Clusterplattformen selbst mit Plattformen aus anderen Regionen und Ländern und führen gemeinsame Veranstaltungen durch (CP-Bahn). Darüber hinaus gibt es teilweise Informationsveranstaltungen über externe Märkte und bei Interesse regen sie zu Delegationsreisen an, die dann unter der Federführung von „Bayern International" durchgeführt werden (CP-Nano). Die Förderung von globalen Pipelines wird neben den praktischen Problemen zudem häufig vom fehlenden Interesse der Unternehmen an diesem Thema erschwert. So sehen die Clustermanager in der fehlenden globalen Vernetzung der von ihnen betreuten Unternehmen teilweise durchaus ein Entwicklungshemmnis für ihren Cluster (CP-IKT). Solange die Unternehmen jedoch selbst dieses Problem nicht erkennen (wollen), sind den Clustermanagern die Hände gebunden. Um Wissen über und Kontakte zu externen Akteuren zu erlangen, binden die Plattformen ferner international gut vernetzte Personen und Organisationen in ihre Strukturen und Maßnahmen ein. Dabei sind sie allerdings von der Bereitschaft dieser Personen abhängig, ihr Wissen und ihre Kontakte mit anderen zu teilen.

Die Clusterplattformen zeigen damit eine klare Tendenz, die Förderung der internen (institutionellen) Clusterdimen- 
sion zu bevorzugen. Während nahezu jede Handlung der Plattformen darauf ausgelegt ist, regionale Akteure zur $\mathrm{Zu}$ sammenarbeit anzuregen, haben sie Probleme damit, externe Akteure in ihre Maßnahmen einzubinden. Problematisch ist insbesondere, dass die Arbeit der Plattformen die Transaktionskosten von regionsinternen Kooperationen senken können, während ihnen nur eingeschränkte Mittel zur Verfügung stehen, die Kosten beim Aufbau globaler Pipelines zu verringern. Steht ein Unternehmen vor der Frage, ob es mit einem lokalen oder mit einem externen Partner zusammenarbeiten soll, verleiten es die Clusterplattformen dazu, den lokalen Partner zu wählen. Langfristig kann diese Tendenz den Cluster von wichtigen Innovationsimpulsen abschneiden und zu gefährlichen Sperren (lock-ins) führen. Diese Ausrichtung nach innen ist ein grundsätzliches Problem der endogenen Wirtschaftsförderung, das in der Clusterförderung besonders deutlich wird (vgl. z. B. Lagendijk/ Pijpers 2013).

Aus der Perspektive der wissensbasierten Clustertheorie (vgl. Malmberg/Maskell 2002), können die Plattformen Wissen im Cluster dementsprechend besser zusammenführen und es weniger gut diversifizieren. Die Verstärkung der regionsinternen Interaktionen durch die Plattformen erleichtert Wissenstransfers und damit (kurzfristige) Innovationsgewinne. Für den langfristigen Erfolg eines Clusters ist jedoch auch eine fortlaufende Erneuerung und Diversifizierung der lokalen Wissensbasis notwendig. Dazu sind vor allem Kontakte zu externen Akteuren wichtig, die die Plattformen jedoch nur schwer herstellen können. Allerdings suchen die Plattformen ständig selbst neue Themen und Trends, die für die von ihnen geförderten Akteure interessant sein könnten und die sie auf den Veranstaltungen präsentieren. Darüber hinaus kann die Förderung der Plattformen theoretisch zur Verbesserung der Aufnahmekapazität (absorptive capacity) der Clusterakteure beitragen und somit deren Fähigkeiten zur Aufnahme und Verarbeitung von externem Wissen erhöhen (vgl. Cohen/Levinthal 1990).

Interaktionen zwischen Wissenschaft und Wirtschaft. Dieses Thema ist den Clustermanagern sehr wichtig und sie sehen hier einen guten Weg, ihren Cluster innovativer zu machen. Um derartige Interaktionen zu fördern, werden zunächst Akteure aus Wissenschaft und Wirtschaft in die internen Strukturen der Clusterplattformen eingebunden. Beliebt sind auch Veranstaltungen an Universitäten oder Forschungseinrichtungen und Maßnahmen zur Konzeption von Studiengängen. Probleme bei der Förderung dieser Art von Interaktionen ergeben sich vor allem daraus, dass die Unternehmen für sich häufig keinen Bedarf daran erkennen können (CP-IKT, CP-Nano; CP-NW). Gegenüber den Akteuren aus der Wissenschaft fehlen zudem eigene Fördermittel (CP-Ern).
Arbeitsmarkt. Die Clusterplattformen unternehmen einiges zur qualitativen und quantitativen Verbesserung der Angebotsseite des Arbeitsmarktes. Einen Schwerpunkt bilden dabei Qualifikationsmaßnahmen, die meistens zusammen mit anderen Akteuren wie den Industrie- und Handelskammern durchgeführt werden (CP-Ern). Derartige Maßnahmen finden vor allem in nicht technologischen Bereichen statt und sollen beispielsweise kaufmännisches Wissen verbessern. Bei einigen Plattformen werden aber bewusst keine Qualifikationsmaßnahmen unternommen, weil es bereits andere öffentliche und private Anbieter gibt oder kein $\mathrm{Be}$ darf besteht (CP-IKT). Teilweise befürchten die Unternehmen auch, dass sie durch derartige Maßnahmen ihre Mitarbeiter verlieren könnten. Um das quantitative Angebot an Arbeitskräften zu verbessern, gibt es Marketingveranstaltungen, die sich beispielsweise an Schüler und Studenten richten, um sie für die jeweilige Branche zu begeistern (CPNano). Maßnahmen zur Förderung der Arbeitsplatzmobilität finden aufgrund fehlender Bereitschaft der Unternehmen nicht statt (CP-IKT).

Neue Unternehmen. An Bemühungen zur Ansiedlung von externen Unternehmen in Bayern beteiligen sich die Clusterplattformen nur indirekt. Diese Aufgabe wird vor allem von „Invest in Bavaria“ übernommen. Auf Anfrage wird jedoch die Unternehmensansiedlung durch die Vermittlung von Kontakten und Informationen unterstützt. Ferner beteiligen sich die Plattformen am allgemeinen Standortmarketing. Maßnahmen zur Gründerförderung übernehmen die Plattformen nicht, was auch hier wieder daran liegt, dass diese Aufgabe bereits von anderen Förderakteuren übernommen wird. Junge Unternehmen sind bei den Aktivitäten der Plattformen selbstverständlich dennoch willkommen, jedoch fehlen diesen häufig die Kapazitäten zur aktiven Teilnahme. Eine Sonderbehandlung genießen junge Unternehmen nicht (CP-IKT).

Wettbewerb und Rivalität. Trotz der herausragenden Stellung von Wettbewerb und Rivalität in der Clustertheorie nach Porter (1998a: 211 f.; Porter 1998b: 167) wird dieser Aspekt von den Clusterplattformen fast gar nicht gefördert (WissBeo1). Der Hauptgrund dafür ist, dass die Arbeitsweise der Plattformen auf das Finden von Konsens und Ergänzungspotenzialen ausgerichtet ist und eventuelle Konflikte vermieden werden (CP-Ern). Zudem lassen sich Wettbewerb und Rivalität kaum durch Veranstaltungen oder Projekte fördern, wodurch sie sich dem Werkzeugkasten der Plattformen entziehen. Auch eine wettbewerbsmäßige Vergabe der eigenen Förderleistungen ist nicht praktikabel, da die Plattformen in der Regel dann tätig werden, wenn die Unternehmen von sich aus zögern, aktiv zu werden. Wettbewerbe würden die Hemmschwellen nur weiter erhöhen. Die einzigen Maßnahmen, die die Plattformen in diesem 
Bereich unternehmen, sind Innovationspreise, die einige von ihnen ausloben. Diese können zumindest einen psychologischen Anreiz für intensivierten Wettbewerb geben, sollen allerdings hauptsächlich Marketingeffekte erzielen.

Spezialisierte Infrastruktur. Den Aufbau spezialisierter Infrastruktur betreiben die Clusterplattformen selbstverständlich nicht selbst. Falls sich Akteure jedoch mit einem konkreten Bedarf an sie wenden, können sie die Interessen bündeln und an die Politik weitervermitteln. Derartige Handlungen, die zur Einrichtung eines Forschungsinstituts führten, wurden bereits erfolgreich durchgeführt (CPNano). Von sich aus werden die Plattformen jedoch nicht aktiv.

Institutionelle Rahmenbedingungen. Die institutionellen Rahmenbedingungen umfassen formelle Gesetze und informelle Regelsysteme wie Normen und Konventionen. Während letztere kaum direkt förderbar sind und die Plattformen auch keine Versuche in diese Richtung unternehmen (CP-Nano), verhält es sich mit den gesetzlichen Regelungen wie mit der spezialisierten Infrastruktur: Wenn sich Unternehmen oder andere Akteure mit konkreten Interessen zur Veränderung der gesetzlichen Rahmenbedingungen an sie wenden, können sie die Interessen bündeln und an die Politik weitervermitteln. Insbesondere beim Cluster Finanzdienstleistungen, bei dem politische Vorgaben eine bedeutende Rolle spielen, gibt es derartige Lobbyismusaktivität. Solange die Clusterplattformen hierbei den Konsens der Clusterakteure vertreten, wird auch keine Verletzung der Neutralität befürchtet.

Zugang zu Kapital. Zur Förderung der Finanzierungsmöglichkeiten der bayerischen Unternehmen gibt es bereits andere Akteure (LfA Förderbank Bayern; Bayern Kapital). Die Clusterplattformen übernehmen hier bei Bedarf nur die Weitervermittlung. Allerdings bemühen sie sich insbesondere bei den Projekten aktiv um die Gewinnung von weiteren Fördermitteln, auch um den Eigenanteil ihrer Finanzierung zu erhöhen. Der Cluster Finanzdienstleistungen bemüht sich zudem gezielt um eine starke Vernetzung von Finanz- und Realwirtschaft und auch andere Plattformen binden bewusst Akteure aus der Finanzbranche in ihre Clusterstrukturen ein (CP-Fin).

Bekanntheit/Image. Marketingmaßnahmen werden von den Clusterplattformen oft durchgeführt und von der Politik auch eingefordert (Ref-StMWIVT). Das betrifft zum einen das Standortmarketing nach außen, um Bayern als Stand-, Geschäfts- oderArbeitsort attraktiv erscheinen zu lassen, aber auch Maßnahmen, um die Produkte der Clusterakteure in und außerhalb Bayerns stärker bekannt zu ma- chen. Da viele Akteure Interesse daran haben, gibt es bei diesen Maßnahmen keine Probleme.

Nachfragebedingungen. Spezialisierte Nachfragebedingungen können selbstverständlich nicht direkt durch Maßnahmen von Clusterplattformen erzeugt werden. Eine Förderung wäre theoretisch durch eine dezidierte Leitmarktpolitik unter Einbindung der Plattformen seitens der Regierung(en) denkbar. Diese können beispielsweise durch gezielte Kommunikation und Kooperationsanbahnung dazu beitragen, die Unternehmensaktivität auf die angestrebten Leitmärkte zu fokussieren. Derartige Aktivität war bisher allerdings nicht festzustellen.

\section{Zusammenfassung}

In der Gesamtbetrachtung wird deutlich, dass die meisten Clustermechanismen in der Praxis gefördert werden können. Tab. 4 stellt die Ergebnisse der Untersuchung für die einzelnen Clustermechanismen dar und zeigt, welche institutionellen und strukturellen Faktoren die Förderung behindern oder begünstigen. Darüber hinaus werden als Ergänzung zu den strukturellen Faktoren die Akteure benannt, die im gesamten Fördersystem für die Förderung der jeweiligen Mechanismen zuständig sind. Die Auswahl orientiert sich am bayerischen Fördersystem, erhebt aber aufgrund der großen Vielfalt von konkreten Fördersystemen keinen Anspruch auf Vollständigkeit.

Für die Clusterplattformen sind institutionelle Faktoren in ihrem Umfeld grundsätzlich von großer Bedeutung. Auf allgemeiner Ebene führen sie dazu, dass die Maßnahmen der Clusterplattformen sehr kontextspezifisch sind. Dafür sorgt insbesondere die Erwartung, dass die Plattformen den Unternehmen einen konkreten Nutzen bringen. Während der Clusterpolitik auf der konzeptionellen Ebene häufig (zu Recht) vorgeworfen wird, dass lokale Kontexte nicht ausreichend berücksichtigt werden (Bergman/Feser 1999; Asheim/Cooke/Martin 2006; Kiese 2008b; Kiese 2012; Benner 2012; Fromhold-Eisebith 2014), gilt dies für die Umsetzungsebene nicht mehr. Hier passen sich die Clusterplattformen stark an die lokalen Strukturen an (z. B. im Hinblick auf die Bedeutung von horizontalen oder vertikalen Clusterstrukturen). Die auf der konzeptionellen Ebene häufig geäuBerte Kritik, dass Clusterpolitik oft auf kurzfristige Gewinne setzt (z. B. Kiese 2008b: 133; Kiese 2012: 326), gilt auf der praktischen Ebene allerdings genauso. Auch hier führt die Erwartung kurzfristiger Gewinne (diesmal von Seiten der Unternehmen) dazu, dass die Clusterentwicklung einer gewissen Kurzsichtigkeit unterliegt. Das Problem ist hierbei allerdings weniger, dass der ,lange Atem` fehlt, sondern dass unter Umständen wichtige ökonomische Entwicklungen verpasst werden, weil man sich nur auf das Bekannte 
Tab. 4 Institutionelle und strukturelle Einschränkungen bei der Förderung der Clustermechanismen

\begin{tabular}{|c|c|c|c|}
\hline Clustermechanismus & $\begin{array}{l}\text { Institutionelle Einschränkungen aus der } \\
\text { Sicht der Clusterplattformen }\end{array}$ & $\begin{array}{l}\text { Strukturelle Einschränkungen aus } \\
\text { der Sicht der Clusterplattformen }\end{array}$ & $\begin{array}{l}\text { Organisationale Zuständigkeiten } \\
\text { (Auswahl) }\end{array}$ \\
\hline \multicolumn{4}{|l|}{ Interaktion } \\
\hline \multicolumn{4}{|l|}{ Nach Art der Interaktion } \\
\hline Stand-by-Kontakte & Keine Einschränkungen & $\begin{array}{l}\text { Kaum Einschränkungen; Überblick } \\
\text { zu wahren ist teilweise schwierig }\end{array}$ & Clusterplattformen, Verbände \\
\hline $\begin{array}{l}\text { Rauschen, Cafeteria- } \\
\text { Effekte }\end{array}$ & Keine Einschränkungen & Keine Einschränkungen & Clusterplattformen, Verbände \\
\hline $\begin{array}{l}\text { Communities of Prac- } \\
\text { tice }\end{array}$ & Keine Einschränkungen & $\begin{array}{l}\text { Positiv: Werden in den Organisati- } \\
\text { onsstrukturen der Clusterplattformen } \\
\text { verankert }\end{array}$ & Clusterplattformen, Verbände \\
\hline Konkrete Kooperation & $\begin{array}{l}\text { Teilweise Einschränkung durch Angst, } \\
\text { Wissen zu verlieren. Positiv: Verspre- } \\
\text { chen direkte Gewinne; Neutralität der } \\
\text { Plattformen ermöglicht Kooperationen, } \\
\text { die sonst nicht möglich wären }\end{array}$ & $\begin{array}{l}\text { Kaum Einschränkungen; Suche nach } \\
\text { Partnern kann schwierig sein }\end{array}$ & Clusterplattformen \\
\hline \multicolumn{4}{|c|}{ Nach Interaktionspartner } \\
\hline Vertikal - horizontal & $\begin{array}{l}\text { Bei horizontalen Beziehungen teilweise } \\
\text { Einschränkung durch Angst, Wissen zu } \\
\text { verlieren }\end{array}$ & $\begin{array}{l}\text { Positiv: Clusterplattformen passen } \\
\text { ihre Maßnahmen gut an vorhandene } \\
\text { vertikale bzw. horizontale Strukturen } \\
\text { an }\end{array}$ & Nicht zutreffend \\
\hline Intern - extern & $\begin{array}{l}\text { Teilweise fehlt Clustermitgliedern } \\
\text { Bewusstsein für Potenziale; durch } \\
\text { Politik ist Fokus auf regionale Akteure } \\
\text { angelegt }\end{array}$ & $\begin{array}{l}\text { Zugang zu externen Kooperations- } \\
\text { partnern ist schwierig }\end{array}$ & $\begin{array}{l}\text { Clusterplattformen, Ansied- } \\
\text { lungs-, Exportförderung }\end{array}$ \\
\hline $\begin{array}{l}\text { Wissenschaft - Wirt- } \\
\text { schaft }\end{array}$ & $\begin{array}{l}\text { Teilweise fehlt Clustermitgliedern } \\
\text { Bewusstsein für Potenziale }\end{array}$ & $\begin{array}{l}\text { Zur stärkeren Einbindung der Wis- } \\
\text { senschaft fehlen den Clusterplattfor- } \\
\text { men eigene Fördermittel }\end{array}$ & $\begin{array}{l}\text { Clusterplattformen, Wissen- } \\
\text { stransfer-Stellen u. a. }\end{array}$ \\
\hline \multicolumn{4}{|l|}{ Weitere Faktoren } \\
\hline \multicolumn{4}{|l|}{ Arbeitsmarkt } \\
\hline Quantitativ & Keine Einschränkungen & $\begin{array}{l}\text { Größe des Arbeitsmarktes lässt sich } \\
\text { kaum und wenn nur indirekt beein- } \\
\text { flussen }\end{array}$ & $\begin{array}{l}\text { Allgemeine Arbeitsmarktpoli- } \\
\text { tik; Clusterplattformen können } \\
\text { durch Marketingmaßnahmen } \\
\text { unterstützen }\end{array}$ \\
\hline Qualitativ & $\begin{array}{l}\text { Teilweise Einschränkungen durch } \\
\text { Angst, Wissen zu verlieren }\end{array}$ & $\begin{array}{l}\text { Konkurrenz zu anderen Förderakteu- } \\
\text { ren, daher häufig Kooperation }\end{array}$ & $\begin{array}{l}\text { Allgemeine Bildungspolitik, } \\
\text { Kammern, weitere Bildungs- } \\
\text { angebote, Clusterplattformen } \\
\text { können unterstützen }\end{array}$ \\
\hline Mobilität & $\begin{array}{l}\text { Förderung wird durch die Angst } \\
\text { der Unternehmen, ihr Wissen zu } \\
\text { verlieren, weitgehend verhindert }\end{array}$ & Wird nicht gefördert & $\begin{array}{l}\text { Allgemeine Arbeitsmarktpolitik } \\
\text { (z. B. Kündigungsschutz) }\end{array}$ \\
\hline
\end{tabular}

konzentriert, das vor einem liegt. Die Clusterplattformen erkennen das Problem durchaus und versuchen gegenzusteuern, indem sie die Maßnahmen auf bestimmte ,Megatrends“ wie den demographischen Wandel ausrichten (CP-NW).

Im Hinblick auf die einzelnen Clustermechanismen gibt es durch institutionelle Faktoren nur in wenigen Fällen eine tatsächliche Einschränkung für die Förderaktivität. Zu nennen sind hier vor allem die Arbeitsplatzmobilität und der Wettbewerb, die durch Clusterplattformen nicht gefördert werden können, da Tätigkeit in diesen Feldern den grundlegenden Erwartungen der Unternehmen widersprechen (CPErn, CP-IKT). Um beides stärker zu fördern, könnten beispielsweise Förderinstrumente etabliert werden, die weniger auf direkte Interaktion der Stakeholder setzen (und da- mit stark durch institutionelle Rahmenbedingungen beeinflusst sind), sondern verstärkt mit Anreizmitteln oder formellen Institutionen arbeiten (z. B. fehlenden Wettbewerb durch Änderung der Wettbewerbspolitik anregen). Das hätte jedoch weitreichende Konsequenzen für andere gesellschaftliche Teilbereiche, sodass genau abzuwägen ist, ob derartige Eingriffe erstrebenswert sind. Die Intensivierung des Wettbewerbs ist unter Umständen auch durch eine gezielte Leitmarktpolitik möglich.

Größere Einschränkungen struktureller Natur liegen nur beim Aufbau von Kooperationen mit externen Partnern vor, da die Plattformen selbst die potenziellen Akteure nur schwer erreichen. Um den sich daraus ergebenden Innenfokus der Clusterförderung abzuschwächen, könnte zum 
Tab. 4 Institutionelle und strukturelle Einschränkungen bei der Förderung der Clustermechanismen (Fortsetzung)

\begin{tabular}{|c|c|c|c|}
\hline Clustermechanismus & $\begin{array}{l}\text { Institutionelle Einschränkungen aus der } \\
\text { Sicht der Clusterplattformen }\end{array}$ & $\begin{array}{l}\text { Strukturelle Einschränkungen aus } \\
\text { der Sicht der Clusterplattformen }\end{array}$ & $\begin{array}{l}\text { Organisationale Zuständigkeiten } \\
\text { (Auswahl) }\end{array}$ \\
\hline \multicolumn{4}{|l|}{ Neue Unternehmen } \\
\hline Externe & Nicht zutreffend & $\begin{array}{l}\text { Förderung durch andere Akteure; } \\
\text { Zugang zu externen Kooperations- } \\
\text { partnern ist schwierig }\end{array}$ & $\begin{array}{l}\text { Ansiedlungsförderung (Bayern } \\
\text { International, Invest in Bavaria) }\end{array}$ \\
\hline Start-ups/Spinn-offs & Nicht zutreffend & $\begin{array}{l}\text { Förderung durch andere Akteure; } \\
\text { jungen Unternehmen fehlen Kapa- } \\
\text { zitäten, um sich an Maßnahmen der } \\
\text { Clusterplattformen zu beteiligen }\end{array}$ & $\begin{array}{l}\text { Verschiedene Akteure der Grün- } \\
\text { derförderung }\end{array}$ \\
\hline \multicolumn{4}{|l|}{ Wettbewerb/Rivalität } \\
\hline- & $\begin{array}{l}\text { Förderung wird durch Gewinnori- } \\
\text { entierung der Unternehmen weitge- } \\
\text { hend verhindert }\end{array}$ & $\begin{array}{l}\text { Wegen starker Spezialisierung der } \\
\text { Unternehmen gibt es häufig kaum } \\
\text { direkte Konkurrenz }\end{array}$ & $\begin{array}{l}\text { Allgemeine Wettbewerbspolitik, } \\
\text { Leitmarktpolitik }\end{array}$ \\
\hline \multicolumn{4}{|c|}{ Spezialisierte Infrastruktur } \\
\hline- & Keine Einschränkungen & $\begin{array}{l}\text { Nur Weitervermittlung an zuständige } \\
\text { Akteure (lobbying) }\end{array}$ & $\begin{array}{l}\text { Allgemeine Technologie-, For- } \\
\text { schungspolitik, Allgemeine } \\
\text { Raumplanung }\end{array}$ \\
\hline \multicolumn{4}{|c|}{ Institutionelle Rahmenbedingungen } \\
\hline Formell & $\begin{array}{l}\text { Wenig Einschränkungen; Neutralität } \\
\text { muss gewahrt werden }\end{array}$ & $\begin{array}{l}\text { Nur Weitervermittlung an zuständige } \\
\text { Akteure (Lobbying) }\end{array}$ & Allgemeine Gesetzgebung \\
\hline Informell & Nicht zutreffend & $\begin{array}{l}\text { Nicht zutreffend (soziale Institutio- } \\
\text { nen können kaum geplant, geändert } \\
\text { oder erzeugt werden) }\end{array}$ & Nicht zutreffend \\
\hline \multicolumn{4}{|l|}{ Zugang zu Kapital } \\
\hline- & Keine Einschränkungen & $\begin{array}{l}\text { Nur Weitervermittlung an zuständige } \\
\text { Akteure }\end{array}$ & $\begin{array}{l}\text { Verschiedene öffentliche und } \\
\text { private Finanzmarktakteure } \\
\text { (z. B. LfA Förderbank Bayern, } \\
\text { Bayern Kapital); verschiedene } \\
\text { Förderprogramme }\end{array}$ \\
\hline \multicolumn{4}{|l|}{ Bekanntheit/Image } \\
\hline- & Keine Einschränkungen & Keine Einschränkungen & $\begin{array}{l}\text { Allgemeines Standortmarketing, } \\
\text { Unterstützung durch Cluster- } \\
\text { plattformen }\end{array}$ \\
\hline \multicolumn{4}{|l|}{ Nachfragebedingungen } \\
\hline- & Keine Einschränkungen & $\begin{array}{l}\text { Können nur schwer erzeugt wer- } \\
\text { den; Thema wird bisher von den } \\
\text { Clusterplattformen wenig beachtet }\end{array}$ & Leitmarktpolitik \\
\hline
\end{tabular}

Fett Bedeutende Einschränkung, welche eine effektive Förderung weitgehend verhindert

Beispiel bei allen Clusteraktivität eine Beteiligung externer Akteure eingefordert werden. Das würde den Aufwand der Förderung zwar deutlich erhöhen, aber: ,[i]n order to generate and maintain competitiveness [...], it is crucial to identify and acquire appropriate knowledge pools that exist beyond easily accessible ,comfort zones"“ (Bathelt/ Cohendet 2014: 871). Die bereits begonnene Vernetzung der Clusterplattformen mit externen Förderakteuren (crossclustering), die verstärkte Einbindung von extern gut vernetzten Akteuren oder sogar von Personen aus anderen Regionen in die Organisationsstrukturen erscheinen als die besten Wege, dieses Defizit der Clusterförderung zukünftig zu beseitigen.

Des Weiteren können einige Clustermechanismen generell nicht oder nur stark eingeschränkt und extrem langfris- tig gefördert werden. Das gilt insbesondere für informelle Institutionen oder die spezialisierten Nachfragebedingungen. Den Clusterplattformen fehlen zudem bei einer Reihe von Mechanismen die entsprechenden Förderinstrumente, die dafür häufig bei anderen Förderakteuren liegen. Die Clusterplattformen konzentrieren sich generell auf die Förderung von Interaktionen bzw. Netzwerken, während die meisten anderen Clustermechanismen von anderen Akteuren bearbeitet werden. Eine umfassende Clusterförderung ,aus einem Guss" ist daher nur mit Abstrichen zu erkennen. Grundsätzlich muss das nicht problematisch sein. Thematisch orientierte Förderbereiche wie die Ansiedlungsund Gründerförderung oder die Verbesserung der Finanzierungsmöglichkeiten für lokale Unternehmen werden aus gutem Grund von spezialisierten Akteuren betreut. Die Si- 
tuation verdeutlicht vielmehr, dass an den Schnittstellen zwischen sektoral und thematisch orientierter Förderung ein Vermittlungsproblem entstehen kann. Der sektoralen Clusterförderung stehen nicht alle notwendigen Instrumente zur Verfügung, während die thematisch spezialisierten Akteure sektorale Besonderheiten und Bedürfnisse nur eingeschränkt kennen. Folglich ist es schwierig, eine umfassende Entwicklungsstrategie zu entwerfen und umzusetzen, die alle Clustermechanismen und ihr Zusammenspiel passend für den jeweiligen regionalen Kontext einbezieht. In der Praxis ignorieren sich die verschiedenen Förderakteure einander zwar nicht gegenseitig, gewisse Reibungsverluste sind dennoch zu erwarten. Dieser Punkt verdeutlicht insbesondere, dass sich eine umfassende Clusterförderung nicht in der Einrichtung von Clusterplattformen erschöpfen sollte. Die größte Herausforderung der Förderpolitik ist daher zunehmend eine sinnvolle strategische Koordination der verschiedenen Förderakteure und -programme.

\section{Literatur}

Asheim, B. T.; Cooke, P.; Martin, R. (2006): The rise of the cluster concept in regional analysis and policy. A critical assessment. In: Asheim, B. T.; Cooke, P.; Martin, R. (Hrsg.): Clusters and regional development. Critical reflections and explorations. London, 1-29.

Bathelt, H.; Cohendet, P. (2014): The creation of knowledge. Local building, global accessing and economic development - toward an agenda. In: Journal of Economic Geography 14, 5, 869-882.

Bathelt, H.; Glückler, J. (2012): Wirtschaftsgeographie. Ökonomische Beziehungen in räumlicher Perspektive. Stuttgart.

Bathelt, H.; Malmberg, A.; Maskell, P. (2004): Clusters and knowledge. Local buzz, global pipelines and the process of knowledge creation. In: Progress in Human Geography 28, 1, 31-56.

Bayerische Staatskanzlei (2006): High-Tech-Offensive Bayern. Bilanz und Perspektiven. München.

Bayerischer Industrie- und Handelskammertag (2006): Identifizierung und Bewertung Regionaler Cluster und Regionaler Innovationsund Produktionssysteme (RIPS) in Bayern. München.

Bayerischer Landtag (2005): Cluster-Strategie der Staatsregierung. Antwort des Staatsministeriums für Wirtschaft, Infrastruktur, Verkehr und Technologie von 30.06.2005 auf eine schriftliche Anfrage des Abgeordneten Dr. Heinz Kaiser SPD vom 13.05.2005. München.

Beck, R. C.; Heinze, R. G.; Schmid, J. (2014a): Zukunft der Wirtschaftsförderung. Perspektiven und Herausforderungen von regionalen Clusterpolitiken. In: Beck, R. C.; Heinze, R. G.; Schmid, J. (Hrsg.): Zukunft der Wirtschaftsförderung. BadenBaden, 23-38.

Beck, R. C.; Heinze, R. G.; Schmid, J. (Hrsg.) (2014b): Zukunft der Wirtschaftsförderung. Baden-Baden.

Benner, M. (2012): Clusterpolitik. Wege zur Verknüpfung von Theorie und politischer Umsetzung. Berlin. = Wirtschaftsgeographie 52.

Benneworth, P.; Charles, D. (2001): Bridging Cluster Theory and Practice. Learning from the Cluster Policy Cycle. In: OECD (Hrsg.): Innovative Clusters. Drivers of National Innovation Systems. Paris, 389-404.

Berger, C. (2002): Technologie- und Innovationspolitik in Bayern. Düsseldorf. = WSI-Diskussionspapier 105.

Bergman, E. M.; Feser, E. J. (1999): Industry Clusters. A Methodology and Framework for Regional Development Policy in the United States. In: OECD (Hrsg.): Boosting Innovation. The Cluster Approach. Paris, 243-268.
Boekholt, P.; Thuriaux, B. (1999): Public Policies to Facilitate Clusters. Background, Rationale and Policy Practices in International Perspective. In: OECD (Hrsg.): Boosting Innovation. The Cluster Approach. Paris, 381-412.

Boschma, R. A.; Wenting, R. (2007): The spatial evolution of the British automobile industry. Does location matter? In: Industrial and Corporate Change 16, 2, 213-238.

Bresnahan, T.; Gambardella, A.; Saxenian, A. (2001): 'Old Economy' Inputs for 'New Economy' Outcomes. Cluster Formation in the New Silicon Valleys. In: Industrial and Corporate Change 10, 4, 835-860.

Bührer, S.; Kroll, H.; Stahlecker, T.; Brandt, T.; Zimmermann, A.; Koschatzky, K.; Baier, E. (2008): Zwischenevaluation der Cluster-Offensive Bayern. Abschlussbericht. Karlsruhe.

Camagni, R. (1991): Local 'milieu', uncertainty and innovation networks. Towards a new dynamic theory of economic space. In: Camagni, R. (Hrsg.): Innovation Networks. Spatial Perspectives. London, New York, 121-144.

Cohen, W. M.; Levinthal, D. A. (1990): Absorptive Capacity. A New Perspective on Learning and Innovation. In: Administrative Science Quarterly 35, 1, 128-152.

Dahl, M.; Østergaard, C. R.; Dalum, B. (2010): Emergence of regional clusters. The role of spinoffs in the early growth process. In: Boschma, R. A.; Martin, R. (Hrsg.): The Handbook of Evolutionary Economic Geography. Cheltenham, 205-220.

DiMaggio, P. J.; Powell, W. W. (1983): The Iron Cage Revisited. Institutional Isomorphism and Collective Rationality in Organizational Fields. In: American Sociological Review 48, 2, 147-160.

Ebbekink, M.; Lagendijk, A. (2013): What's Next in Researching Cluster Policy. Place-Based Governance for Effective Cluster Policy. In: European Planning Studies 21, 5, 735-753.

Feldman, M.; Francis, J.; Bercovitz, J. (2005): Creating a Cluster While Building a Firm. Entrepreneurs and the Formation of Industrial Clusters. In: Regional Studies 39, 1, 129-141.

Ferrary, M.; Granovetter, M. (2009): The role of venture capital firms in Silicon Valley's complex innovation network. In: Economy and Society 38, 2, 326-359.

Fromhold-Eisebith, M. (2014): Erfolgsgeschichte oder Modeerscheinung? Clusterpolitik im Spannungsfeld von Theorie und Praxis. In: Beck, R. C., Heinze, R. G., Schmid, J. (Hrsg.): Zukunft der Wirtschaftsförderung. Baden-Baden, 67-88.

Fromhold-Eisebith, M.; Eisebith, G. (2008a): Clusterförderung auf dem Prüfstand. Eine kritische Analyse. In: Zeitschrift für Wirtschaftsgeographie 52, 1, 79-94.

Fromhold-Eisebith, M.; Eisebith, G. (2008b): Looking Behind Facades: Evaluating Effects of (Automotive) Cluster Promotion. In: Regional Studies 42, 10, 1343-1356.

Gertler, M.S. (2003): Tacit knowledge and the economic geography of context, or The undefinable tacitness of being (there). In: Journal of Economic Geography 3, 1, 75-99.

Gertler, M. S. (2010): Rules of the game. The place of institutions in regional economic change. In: Regional Studies 44, 1, 1-15.

Glaeser, E.; Kallal, H. D.; Scheinkman, J.; Shleifer, A. (1992): Growth in Cities. In: Journal of Political Economy 100, 6, 1126-1152.

Glückler, J. (2005): Making embeddedness work. Social practice institutions in foreign consulting markets. In: Environment and Planning A 37, 10, 1727-1750.

Grabher, G. (2002): Cool Projects, Boring Institutions. Temporary Collaboration in Social Context. In: Regional Studies 36, 3, 205-214.

Häfner, K. (2014): Clusterplattformen in Bayern zwischen Theorie und Praxis. Möglichkeiten und Begrenzungen für eine clustertheoretisch orientierte Wirtschaftsförderung. Würzburg. = Würzburger Geographische Arbeiten 111.

Hasse, R.; Krücken, G. (2005): Neo-Institutionalismus. Bielefeld.

He, S.; MacNeill, S.; Wang, J. (2014): Assessing Overall Network Structure in Regional Innovation Policies. A Case Study of Cluster Policy in the West Midlands in the UK. In: European Planning Studies 22, 9, 1940-1959. 
Hellmann, K.-U. (2006): Organisationslegitimität im Neo-Institutionalismus. In: Senge, K.; Hellmann, K.-U. (Hrsg.): Einführung in den Neo-Institutionalismus. Wiesbaden. 75-88.

Jappe-Heinze, A.; Baier, E.; Kroll, H. (2008): Clusterpolitik: Kriterien für die Evaluation von regionalen Clusterinitiativen. Karlsruhe.

Ketels, C. (2014): Ein Jahrzehnt Clusterpolitik und -forschung. Implikationen für eine moderne, clusterorientierte Wirtschaftsförderung. In: Beck, R. C.; Heinze, R. G.; Schmid, J. (Hrsg.): Zukunft der Wirtschaftsförderung. Baden-Baden, 45-64.

Kiese, M. (2008a): Stand und Perspektiven der regionalen Clusterforschung. In: Kiese, M.; Schätzl, L. (Hrsg.): Cluster und Regionalentwicklung. Theorie, Beratung und praktische Umsetzung. Dortmund, $9-50$.

Kiese, M. (2008b): Mind the Gap. Regionale Clusterpolitik im Spannungsfeld von Wissenschaft, Politik und Praxis aus der Perspektive der Neuen Politischen Ökonomie. In: Zeitschrift für Wirtschaftsgeographie 52, 2-3, 129-145.

Kiese, M. (2010): Policy transfer and institutional learning. An evolutionary perspective on regional cluster policies in Germany. In: Fornahl, D.; Henn, S.; Menzel, M.-P. (Hrsg.): Emerging clusters. Theoretical, Empirical and Political Perspectives on the Initial Stage of Cluster Evolution. Cheltenham, 324-353.

Kiese, M. (2012): Regionale Clusterpolitik in Deutschland. Bestandsaufnahme und interregionaler Vergleich im Spannungsfeld von Theorie und Praxis. Marburg.

Koschatzky, K. (2012): Cluster quo vadis? Neue Förderansätze in der Innovationspolitik. In: Koschatzky, K.; Stahlecker, T. (Hrsg.): Clusterpolitik quo vadis? Perspektiven der Clusterförderung. Stuttgart, 191-206.

Koschatzky, K.; Pöchhacker-Tröscher, G.; Bührer, S.; Stahlecker, T. (2011): Evaluation der Cluster-Offensive Bayern. Abschlussbericht - Kurzversion. Karlsruhe.

Koschatzky, K.; Stahlecker, T. (Hrsg.) (2012): Clusterpolitik quo vadis? Perspektiven der Clusterförderung. Stuttgart.

Krugman, P. (1991): Geography and Trade. Leuven.

Lagendjik, A.; Pijpers, R. (2013): Beyond the Regional Cradle and Policy Trap. Proximity and Embedding as Development Potentialities. In: European Planning Studies 21, 5, 631-636.

Lindqvist, G.; Ketels, C.; Sölvell, Ö. (2013): The Cluster Initiative Greenbook 2.0. Stockholm.

Lissoni, F. (2001): Knowledge codification and the geography of innovation. The case of Brescia mechanical Cluster. In: Research Policy 30, 9, 1479-1500.

Maillat, D. (1998): Vom „Industrial District“ zum innovativen Milieu. Ein Beitrag zur Analyse der lokalisierten Produktionssysteme. In: Geographische Zeitschrift 86, 1, 1-15.

Malmberg, A.; Maskell, P. (2002): The elusive concept of localization economies. Towards a knowledge-based theory of spatial clustering. In: Environment and Planning A 34, 3, 429-449.

Malmberg, A.; Power, D. (2005): (How) Do (Firms in) Clusters Create Knowledge? In: Industry and Innovation 12, 4, 409-431.

Martin, R.; Sunley, P. (2003): Deconstructing clusters. Chaotic concept or policy panacea? In: Journal of Economic Geography 3, 1, 5-35.

Maskell, P.; Malmberg, A. (1999): Localised learning and industrial competitiveness. In: Cambridge Journal of Economics 23, 2, $167-185$.

Mayring, P. (2010): Qualitative Inhaltsanalyse. Grundlagen und Techniken. Weinheim.
Meyer, J. W.; Rowan, B. (1977): Institutionalized Organizations. Formal Structure as Myth and Ceremony. In: American Journal of Sociology 83, 2, 340-363.

MfFW - Ministerium für Finanzen und Wirtschaft Baden-Württemberg (2015): Regionaler Cluster-Atlas Baden-Württemberg. Überblick über clusterbezogene Netzwerke und Initiativen. Stuttgart.

Nauwelaers, C. (2001): Path-Dependency and the Role of Institutions in Cluster Policy Generation. In: Mariussen, Å. (Hrsg.): Cluster Policies - Cluster Development? Stockholm, 93-107. = Nordregio Report 2/2001.

Porter, M.E. (1998a): Clusters and Competition. New Agendas for Companies, Governments, and Institutions. In: Porter, M.E. (Hrsg.): On Competition. Boston, 197-287.

Porter, M.E. (1998b): The Competitive Advantage of Nations. In: Porter, M.E. (Hrsg.): On Competition. Boston, 155-195.

Power, D.; Lundmark, M. (2004): Working through knowledge pools. Labour market dynamics, the transference of knowledge and ideas, and industrial clusters. In: Urban Studies 41, 5-6, $1025-1044$.

Raines, P. (2000): Developing Cluster Policies in Seven European Regions. Glasgow. $=$ Regional and Industrial Policy Research Paper 42 .

Raines, P. (2001): The Cluster Approach and the Dynamics of Regional Policy-Making. Glasgow. = Regional and Industrial Policy Research Paper 47.

Scott, A. J. (1998): Regions and the world economy. The coming shape of global production, competition, and political order. Oxford, New York.

Scott, W. R. (2001): Institutions and Organizations. Thousand Oaks.

StMWIVT - Bayerisches Staatsministerium für Wirtschaft, Infrastruktur, Verkehr und Technologie (2006): Allianz Bayern Innovativ. Eckpunkte bayerischer Clusterpolitik. München.

StMWIVT - Bayerisches Staatsministerium für Wirtschaft, Infrastruktur, Verkehr und Technologie (2012): Cluster-Offensive Bayern. Im Netzwerk zum Erfolg. München.

Sternberg, R. (2014): Regionale Gründerförderung. Gründungsbezogene Rahmenbedingungen und Gründungsaktivitäten am Beispiel der Region Hannover. In: Beck, R. C.; Heinze, R. G.; Schmid, J. (Hrsg.): Zukunft der Wirtschaftsförderung. BadenBaden, 195-228.

Stockinger, D. U. (2010): Handlungsräume und Akteure der Clusterpolitik in den USA. Implementierungsprozesse in North Carolina, Oregon und Pennsylvania aus politisch-ökonomischer und institutioneller Perspektive. Berlin.

Storper, M.; Venables, A. J. (2004): Buzz: Face-to-face contact and the urban economy. In: Journal of Economic Geography 4, 4, $351-370$.

Tacke, V. (2006): Rationalität im Neo-Institutionalismus. Vom exakten Kalkül zum Mythos. In: Senge, K.; Hellmann, K.-U. (Hrsg.): Einführung in den Neo-Institutionalismus. Wiesbaden, 89-101.

Walgenbach, P.; Meyer, R. E. (2008): Neoinstitutionalistische Organisationstheorie. Stuttgart.

Wurpts, K.; Kirchgeorg, M. (2013): Clusterförderung mit der „Gemeinschaftsaufgabe“. Eine Evaluierung von Konzept und Implementierung in Ostdeutschland. In: Raumforschung und Raumordnung $71,5,427-436$. 